\title{
Vertical magnetic field gradient in the photospheric layers of sunspots
}

\author{
Jayant Joshi ${ }^{1,2}$, Andreas Lagg ${ }^{1}$, Johann Hirzberger ${ }^{1}$, Sami K. Solanki ${ }^{1,3}$, and Sanjiv K. Tiwari ${ }^{1,4}$ \\ 1 Max-Planck-Institut für Sonnensystemforschung, Justus-von-Liebig-Weg 3, 37077 Göttingen, Germany \\ 2 Institute for Solar Physics, Department of Astronomy, Stockholm University, AlbaNova University Centre, 10691 Stockholm, \\ Sweden \\ e-mail: jayant.joshi@astro.su.se \\ 3 School of Space Research, Kyung Hee University, Yongin, 446-701 Gyeonggi Do, Republic of Korea \\ ${ }^{4}$ NASA Marshall Space Flight Center, Mail Code ZP 13, Huntsville, AL 35812, USA
}

Received 25 July 2016 / Accepted 30 September 2016

\begin{abstract}
Aims. We investigate the vertical gradient of the magnetic field of sunspots in the photospheric layer.

Methods. Independent observations were obtained with the Solar Optical Telescope/Spectropolarimeter (SOT/SP) on board the Hinode spacecraft and with the Tenrife Infrared Polarimeter-2 (TIP-2) mounted at the German Vacuum Tower Telescope (VTT). We apply state-of-the-art inversion techniques to both data sets to retrieve the magnetic field and the corresponding vertical gradient along with other atmospheric parameters in the solar photosphere.

Results. In the sunspot penumbrae we detected patches of negative vertical gradients of the magnetic field strength, i.e., the magnetic field strength decreases with optical depth in the photosphere. The negative gradient patches are located in the inner and partly in the middle penumbrae in both data sets. From the SOT/SP observations we found that the negative gradient patches are restricted mainly to the deep photospheric layers and are concentrated near the edges of the penumbral filaments. Magnetohydrodynamic (MHD) simulations also show negative gradients in the inner penumbrae, also at the locations of filaments. In the observations and the simulation negative gradients of the magnetic field vs. optical depth dominate at some radial distances in the penumbra. The negative gradient with respect to optical depth in the inner penumbrae persists even after averaging in the azimuthal direction in the observations and, to a lesser extent, in the MHD simulations. If the gradients in the MHD simulations are determined with respect to geometrical height, then the azimuthal averages are always positive within the sunspot (above $\log \tau=0$ ), corresponding to magnetic field increasing with depth, as generally expected.

Conclusions. We interpret the observed localized presence of negative vertical gradient of the magnetic field strength in the observations as a consequence of stronger field from spines expanding with height and closing above the weaker field inter-spines. The presence of the negative gradients with respect to optical depth after azimuthal averaging can be explained by two different mechanisms: the high corrugation of equal optical depth surfaces and the cancellation of polarized signal due to the presence of unresolved opposite polarity patches in the deeper layers of the penumbra.
\end{abstract}

Key words. Sun: magnetic fields - Sun: activity

\section{Introduction}

The vertical gradient of the magnetic field vector in sunspot photospheres has been studied extensively in the past (for reviews see, e.g., Solanki 2003; Borrero \& Ichimoto 2011). Depending on the diagnostic tools, a wide range of values from 1.0 to $4.0 \mathrm{G} \mathrm{km}^{-1}$ for the vertical gradient of the magnetic field strength in sunspot umbrae have been reported (Westendorp Plaza et al. 2001; Mathew et al. 2003; Orozco Suarez et al. 2005; Sánchez Cuberes et al. 2005; Balthasar \& Gömöry 2008; Borrero \& Ichimoto 2011). Positive values indicate an increase in the magnetic field strength with geometrical depth. Studies that display the vertical field gradient as a function of the normalized radius of the sunspot also differ significantly. Westendorp Plaza et al. (2001), Borrero \& Ichimoto (2011), and Orozco Suarez et al. (2005) interpret the observations to say that the sunspot magnetic field forms canopylike structures in the middle and outer penumbrae. In contrast, Mathew et al. (2003), Sánchez Cuberes et al. (2005), and
Balthasar \& Gömöry (2008) merely conclude that the magnetic field increases with depth everywhere in the sunspot. Tiwari et al. (2015) also do not find evidence for such a canopylike structure in the middle and outer penumbra, although they report on a reversed magnetic field gradient in the inner penumbra (i.e., field strength decreasing with depth), and field canopy starting just outside the sunspot penumbra. Consequently there is a need to revisit the question of the behavior of the vertical field gradient in sunspots.

In the present paper we study the radial dependence of the vertical gradient of the magnetic field strength of a sunspot observed on two different days by Tenerife Infrared Polarimeter-2 (TIP-2; Collados et al. 2007) mounted at the Vacuum Tower Telescope (VTT) and of another sunspot observed by the Spectropolarimeter of the Solar Optical Telescope (SOT/SP; Tsuneta et al. 2008; Shimizu et al. 2008; Suematsu et al. 2008; Ichimoto et al. 2008). To retrieve the atmospheric parameters from VTT/TIP-2 data we carry out inversions of Stokes profiles of the Si I 10827.1 $\AA$ and Ca I 10833.4 $\AA$ spectral lines together. 
A\&A 599, A35 (2017)

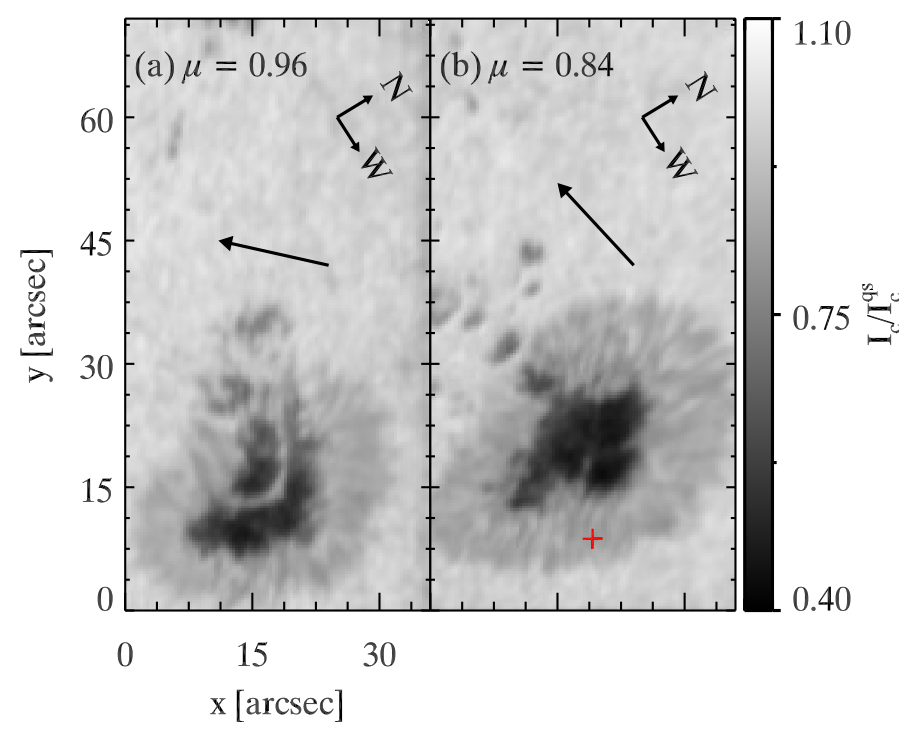

Fig. 1. a) Normalized continuum intensity $I_{\mathrm{c}} / I_{\mathrm{c}}^{q s}$ map of the observed sunspot in AR NOAA 11124 observed on 14 November 2010, obtained from VTT/TIP-2 data. $I_{\mathrm{c}}$ denotes the continuum intensity and $I_{\mathrm{c}}^{\mathrm{qs}}$ is the averaged quiet Sun continuum intensity. b) $I_{\mathrm{c}} / I_{\mathrm{c}}^{\mathrm{qs}}$ map of the same sunspot observed on 16 November 2010. Arrows in both maps indicate the direction to disk center. Solar north and west directions are marked by arrows in the upper right of each panel.

We also use spatially coupled inversions to the Hinode SOT/SP observations (i.e., the Fe I $6301.5 \AA$ and Fe I $6302.5 \AA$ lines) to determine the vertical field gradients. We compare the results from the different data sets with each other and with a 3D magnetohydrodynamic (MHD) simulation of a sunspot by Rempel (2012).

\section{VTT/TIP-2 observations and analysis}

\subsection{Observations}

We observed the leading sunspot in the active region NOAA 11124 on 14 and 16 November 2010. Spectropolarimetric observations were carried out at the VTT/TIP-2. The spectral domain of the observations ranges from $10825 \AA$ to $10835 \AA$ and the spectral sampling corresponds to $10 \mathrm{~m} \AA$ per pixel. The diffraction limit of the VTT at $10830 \AA$ is approximately $0 . " 40$, but owing to moderate seeing conditions the spatial resolution of our data is reduced to $\sim 1$." $^{\prime}$. Scans of the $\sim 36^{\prime \prime}$ wide field of view (FOV) for full Stokes vector measurements require $\sim 20 \mathrm{~min}$. The step size for scanning was set to 0"36 and the pixel size in the slit direction was $0 . " 16$. In order to improve the signal-to-noise ratio we binned four pixels in the direction of dispersion and two pixels in slit direction. The heliocentric coordinates of the centers of the observed FOVs were $\left(12^{\circ} \mathrm{N}, 10^{\circ} \mathrm{W}, \mu=0.96\right)$ on 14 November 2010 and $\left(14^{\circ} \mathrm{N}, 32^{\circ} \mathrm{W}, \mu=0.84\right)$ on 16 November 2010 .

Panels $\mathrm{a}$ and $\mathrm{b}$ of Fig. 1 show the sunspot on 14 and 16 November 2010, respectively, as observed in the continuum intensity at $10833 \AA$. The sunspot shows a light-bridge on 14 November, which disappeared after two days. In general, the sunspot grew and matured in these two days, being more symmetric and simpler on 16 November 2010.

The collected data were treated by applying the standard data reduction steps (Collados 1999, 2003). The continuum level was corrected using a Fourier Transform Spectrometer (FTS) spectrum (Livingston \& Wallace 1991; Wallace et al. 1993).
Table 1. Atmospheric and atomic parameters of the lines used for inversions of the VTT/TIP-2 data.

\begin{tabular}{ccccc}
\hline \hline Line & wavelength $[\AA]$ & $\log (g f)$ & Abundance & $g_{\text {eff }}$ \\
\hline Si I & 10827.1 & 0.210 & 7.549 & 1.5 \\
$\mathrm{Ca}$ I & 10833.4 & 0.058 & 6.360 & 1.5 \\
\hline
\end{tabular}

\subsection{SPINOR inversion of the Si I and $\mathrm{Ca}$ I lines}

To analyze the photospheric properties of the sunspot's magnetic field we apply inversions of Stokes profiles of the Si I $10827.1 \AA$ and Ca I $10833.4 \AA$ lines to satisfy the radiative transfer equation (RTE) under the assumption of local thermodynamic equilibrium (LTE).

The combination of the strong $\mathrm{Si}$ I line and the weak $\mathrm{Ca}$ I line puts more constraints on the free parameters in the inversion process and gives more reliable atmospheres than inversion of a single line, especially when investigating the height stratification of the atmosphere. The employed line parameters of both lines are given in Table 1 . The abundance of Si and $\log (g f)$ of the Si I $10827.1 \AA$ line is inferred from Shchukina et al. (2012) and Shi et al. (2008), respectively. The abundance of $\mathrm{Ca}$ is inferred from Grevesse \& Sauval (1998). We estimated the value of $\log (g f)$ for the Ca I $10833.4 \AA$ line by fitting the quiet Sun profile observed with the FTS (Livingston \& Wallace 1991; Wallace et al. 1993) using the HSRASP atmosphere (Chapman 1979). The quiet Sun spectrum obtained by averaging 255 quiet Sun pixels observed on 14 November 2010 is shown in Fig. 2. To avoid undue influence of the weak Ti I blend at $10833.66 \AA$ we restricted the inversion of the $\mathrm{Ca}$ I line to the wavelength range 10833.08 to $10833.52 \AA$.

Bard \& Carlsson (2008) have shown that the line core of Si I $10827.1 \AA$ is affected by non-local thermodynamic equilibrium (NLTE) conditions. They demonstrated that the line core intensity of Si I $10827.1 \AA$ under NLTE conditions is lower than the line core intensity under LTE conditions. Kuckein et al. (2012) studied the effect of neglecting NLTE effects on atmospheric parameters inferred from inversions. They found that the most affected parameter is the temperature, while the influence on the magnetic field vectors and velocities are negligible. Since in this study the temperature stratification is of minor relevance, we use the simpler LTE approach for our inversions.

To retrieve the atmospheric parameters in the photosphere, we used the SPINOR inversion code (Frutiger et al. 1999, 2000; van Noort 2012), which is based on the STOPRO routines (Solanki 1987). The initial model atmosphere consists of a magnetic field strength, $B$, which varies linearly with the logarithm to the power of 10 of the optical depth $(\log \tau)$. In other words, it is similar to an atmosphere with two nodes for the magnetic field strength if the two nodes are the lowest $(\log \tau=0.0)$ and highest $(\log \tau=-2.3)$ nodes in our inversion. Hence, the magnetic field has two degrees of freedom. Three nodes for the line-ofsight (LOS) velocity, $v_{\text {los }}$, and the temperature, $T$, were used. The other atmospheric parameters such as inclination of the magnetic field relative to LOS, $\gamma$; its azimuth direction, $\phi$; and the micro-turbulent velocity, $v_{\text {mic }}$, were assumed to be constant with height. The three nodes used are at $\log \left(\tau_{630}\right)=0.0,-0.7$ and -2.3 , where $\tau_{630}$ corresponds to the optical depth at $630 \mathrm{~nm}^{1}$. The nodes were selected by looking at magnetic field response

1 This reference wavelength for optical depth $\tau$ was chosen to be $630 \mathrm{~nm}$ throughout this paper to facilitate comparison with Hinode SOT/SP observations. 


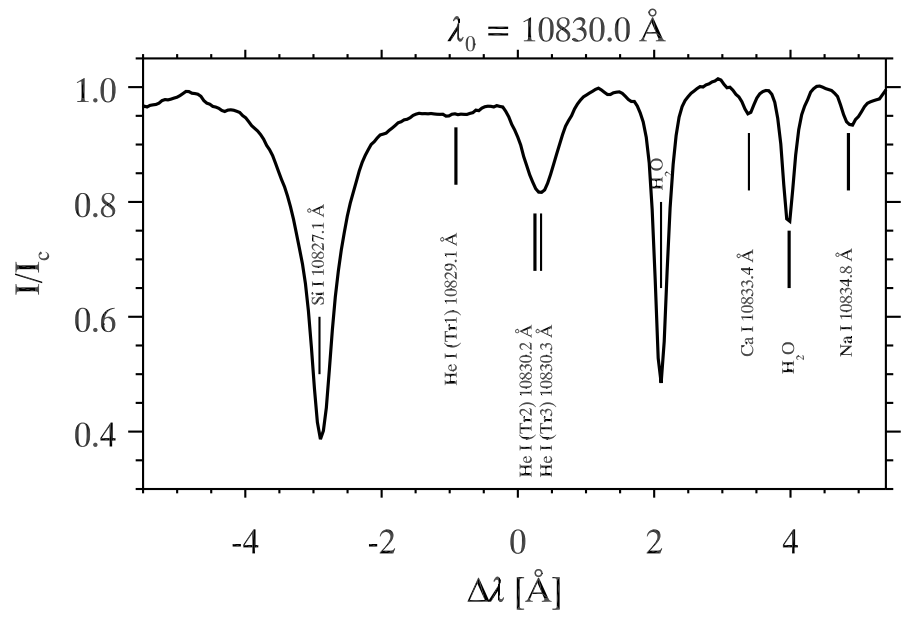

Fig. 2. Averaged quiet Sun spectrum obtained with the VTT/TIP-2 on 14 November 2010. $\Delta \lambda=\lambda-\lambda_{0}$, where $\lambda_{\mathrm{o}}=10830.0 \AA$.
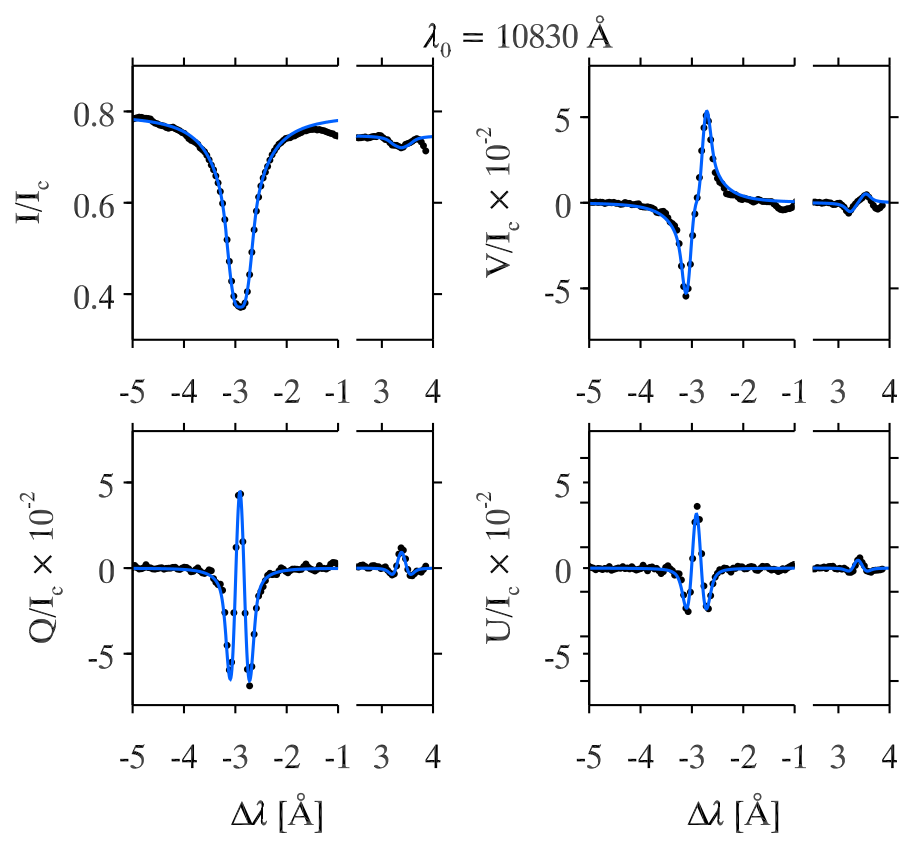

Fig. 3. Best-fit SPINOR inversions of sample Stokes profiles observed by VTT/TIP-2: Dotted black curves represent observed Stokes profiles and solid blue curves represent best fits. The spatial position of the set of Stokes profiles shown here is marked by a red plus sign in Fig. 1b.

functions, $\mathrm{RF}_{B}$. Joshi (2014) presented $\mathrm{RF}_{B}$ of the $\mathrm{Si} \mathrm{I}$ and $\mathrm{Ca}$ I lines for an umbral and different penumbral atmospheres inferred from the MHD simulation of a sunspot by Rempel (2012) (see Figs. 4.3 and 4.5 in chap. 4 of Joshi 2014). They showed that these lines have large values of $\mathrm{RF}_{B}$ at $\log \tau=-0.7$ and -2.3 and non-zero values at $\log \tau=0.0$. An example of observed Stokes profiles and best-fit Stokes profiles through SPINOR inversions is presented in Fig. 3. In general, the match between synthesized and observed profiles is very good.

\subsubsection{Influence of stray light}

To examine the effect of stray light on the retrieved magnetic field vector and on the vertical gradient of $B$, we fitted the same atmospheric model with a second atmospheric component, representing the contribution of global stray light. By global stray light we mean that the stray light function does not vary spatially

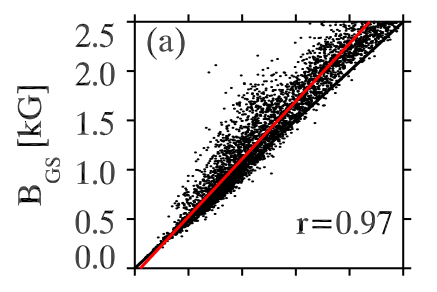

$\begin{array}{llllll}0.0 & 0.5 & 1.0 & 1.5 & 2.0 & 2.5\end{array}$ $\mathrm{B}[\mathrm{kG}]$

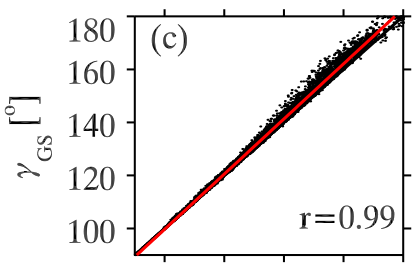

100120140160180 $\gamma\left[{ }^{\mathrm{O}}\right]$
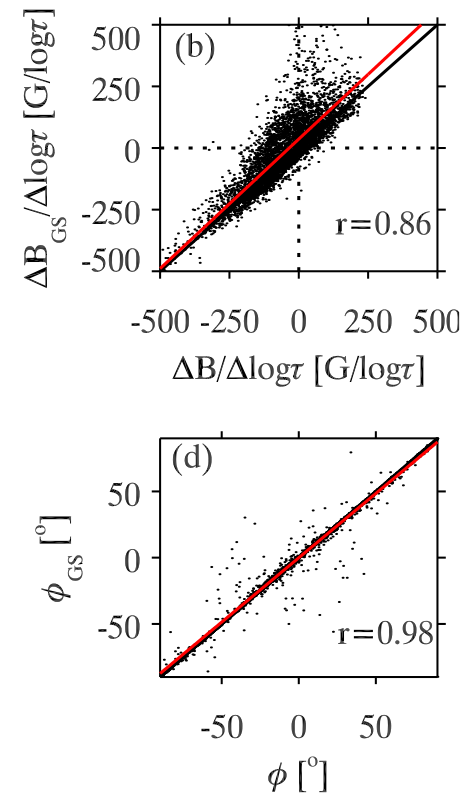

Fig. 4. Scatter plots of the magnetic field components obtained from SPINOR inversions without considering stray light vs. those with stray light taken into account. The plotted data were obtained on 14 November 2010, with VTT/TIP-2. Panels a)-c); and d) show $B, \Delta B / \Delta \log \tau, \gamma$, and $\phi$, respectively. The red line in each panel represents the linear regression. The correlation coefficient, $r$, is shown in the lower right corner of each panel.

in the observed FOV. It is assumed to originate from the broad wings of the point-spread function, typical for seeing-affected ground-based observations, and therefore resembling the shape of the averaged quiet Sun Stokes I profile. The stray light contribution is denoted by $\alpha$ in Eq. (1):

$I_{O}=(1-\alpha) I_{L}+\alpha I_{G S}$.

Here $I_{\mathrm{o}}$ is the observed Stokes $I, I_{\mathrm{L}}$ represents Stokes $I$ of the local component, and $I_{\mathrm{GS}}$ is Stokes $I$ of the stray light component; $\alpha$ is an additional free parameter in the inversions. We are aware that this is a simplified approach that neglects that the absolute amount of stray light might decrease with distance from the quiet Sun and that in the penumbra polarized stray light is probably important as well. This approach is taken purely for test purposes since the fits of the synthesized Stokes profiles to the observed Stokes profiles are very good, even without any consideration of stray light.

A comparison of the obtained magnetic field vectors inferred with and without stray light is presented in Fig. 4, showing very good correlation. Among all the parameters, the vertical gradient displays the fewest correlations (86\%). Although $B$ is somewhat underestimated (by $6 \%$ in the umbra) when stray light is not considered, we see negative and positive vertical gradients of $B$ in both approaches to the inversion. This test suggests that neglecting stray light does not affect the qualitative structure of the vertical gradient of the magnetic field in the sunspot, although there are some quantitative differences. For the analysis presented in this section we therefore use the parameters retrieved from inversions neglecting the stray light contribution.

For the Hinode/SOT data the spatial PSF is constant and well known, and therefore the influence of this spatially inhomogeneous stray light is taken into account (for details see van Noort 2012; van Noort et al. 2013, and Sect. 3). 

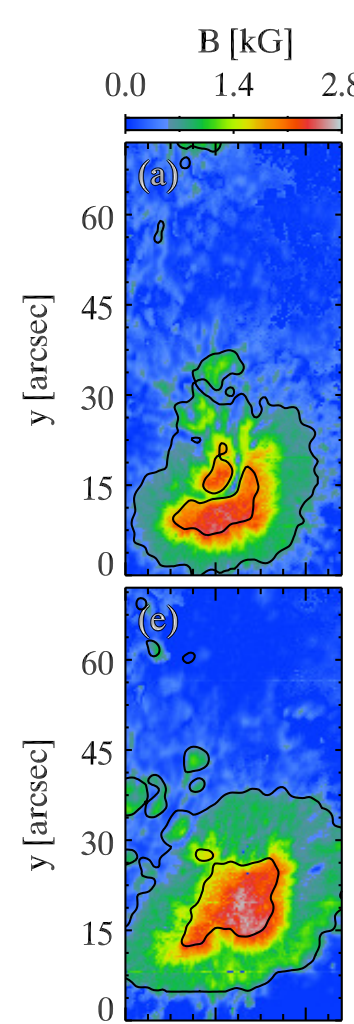

0
$\Delta \mathrm{B} / \Delta \log \tau[\mathrm{G} / \log \tau]$ $2.8-600-300 \quad 0 \quad 300 \quad 0$
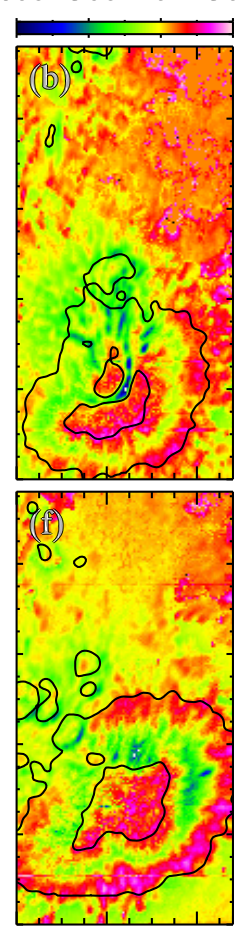$$
0
$$

$$
\mathbf{x}[\operatorname{arcsec}]
$$
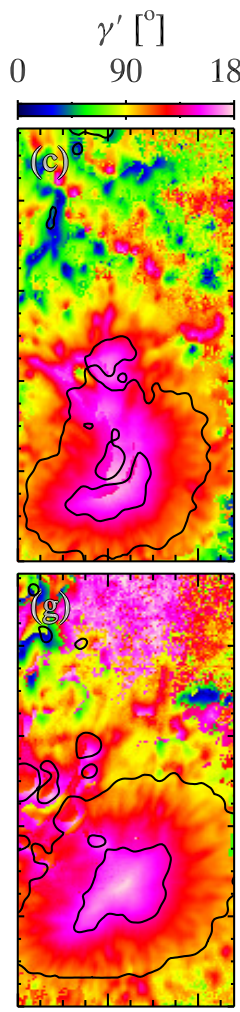

$\begin{array}{lcr}0 & 15 \quad 30 \\ & \mathbf{x}[\operatorname{arcsec}]\end{array}$
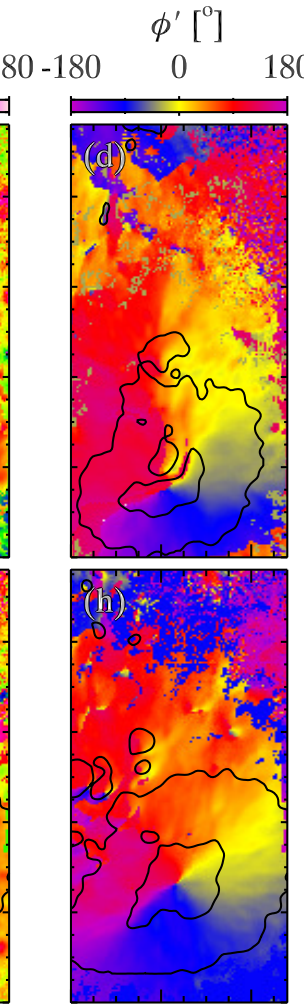

$\begin{array}{lll}0 & 15 & 30\end{array}$

$\mathrm{x}[\operatorname{arcsec}]$
180

\subsection{Inversion results}

The magnetic field parameters retrieved from inversions of the observations from 14 November 2010 are presented in the upper row of Fig. 5. Panels a and b display maps of $B$ and its linear gradient, $(\Delta B / \Delta \log \tau)_{0.0,-2.3}$ of the observed FOV, respectively. The definition of $(\Delta B / \Delta \log \tau)$ is given by

$\left(\frac{\Delta B}{\Delta \log \tau}\right)_{\mathrm{a}, \mathrm{b}}=\frac{(\Delta B)_{\mathrm{a}, \mathrm{b}}}{(\Delta \log \tau)_{\mathrm{a}, \mathrm{b}}}=\frac{B(b)-B(a)}{b-a}$,

where $a$ and $b$ denote lower and upper $\log \tau$ surfaces, respectively. Inclination and azimuth angles of the magnetic field vectors, $\boldsymbol{B}$, are presented in panels c and d of Fig. 5. The magnetic field vectors presented here are projected to disk center coordinates using the transformation matrix given by Wilkinson et al. (1989). The $180^{\circ}$ ambiguity in azimuth direction is resolved by the "acute angle" method (Sakurai et al. 1985; Cuperman et al. 1992). Inclination and azimuth angle of $\boldsymbol{B}$ after correction to the solar disk center coordinates are denoted by $\gamma^{\prime}$ and $\phi \prime$, respectively. Magnetic field parameters obtained from the observations recorded on 16 November 2010 are shown in the lower row of Fig. 5. The umbral and penumbral boundaries shown here, as well as those for the SOT/SP observations and MHD simulation (presented in Sects. 3 and 4 respectively), are determined by the method of cumulative histogram of the continuum intensity as demonstrated by Mathew et al. (2007).

On 14 November the maximum of $B$ amounts to $\sim 2500 \mathrm{G}$ and appears in the darkest part of the umbra; two days later it increases to $\sim 2800 \mathrm{G}$. Maps of $(\Delta B / \Delta \log \tau)_{0.0,-2.3}$ show consistent results on both days: in the umbra $(\Delta B / \Delta \log \tau)_{0.0,-2.3}$ is positive, i.e., $B$ increases with optical depth. In the undisturbed part of the inner and middle penumbra the gradient maps show a ring-like

structure where $(\Delta B / \Delta \log \tau)_{0.0,-2.3}$ is negative, i.e., $B$ decreases with optical depth. In the outer penumbra $(\Delta B / \Delta \log \tau)_{0.0,-2.3}$ is positive, and just outside the visible boundary of the sunspot $(\Delta B / \Delta \log \tau)_{0.0,-2.3}$ is negative again.

The magnetic field response functions, $\mathrm{RF}_{B}$, of Stokes $I$ and $V$ of the spectral line diagnosed here show that $\log \tau$ nodes used in the inversions roughly cover the range of heights to which the lines are sensitive. Figure 6 displays $\mathrm{RF}_{B}$ for two different locations in the penumbra with different sign of $(\Delta B / \Delta \log \tau)_{0.0,-2.3}$. The $\mathrm{RF}_{B}$ for a penumbral atmosphere where $B_{\log \tau=0.0}=833 \mathrm{G}$, $(\Delta B / \Delta \log \tau)_{0.0,-2.3}=57 \mathrm{G} / \log \tau$, and $T_{\log \tau=0.0}=6273 \mathrm{~K}$ are presented in Figs. $6 \mathrm{a}-\mathrm{d}$, while Figs. $6 \mathrm{e}-\mathrm{h}$ show $\mathrm{RF}_{B}$ for an atmosphere where $B_{\log \tau=0.0}=930 \mathrm{G},(\Delta B / \Delta \log \tau)_{0.0,-2.3}=$ $-173 \mathrm{G} / \log \tau$, and $T_{\log \tau=0.0}=6328 \mathrm{~K}$. Wavelength-integrated $\left|R_{B}\right|$ are plotted in Figs. 6i and j, showing that these lines have strong $\mathrm{RF}_{B}$ at $\log \tau=-0.7$ and -2.3 and non-zero values at $\log \tau=0.0$ irrespective of the nature of $(\Delta B / \Delta \log \tau)_{0.0,-2.3}$. The model atmosphere in our inversions consists of a magnetic field strength which varies linearly with $\log \tau$.

To study the radial dependence of the properties of the sunspot atmosphere, we use azimuthal averages of all parameters. The azimuthal averages are computed along 25 isothermal contours obtained from a smoothed temperature map at $\log \tau=0.0$ (see panels a and b of Fig. 7). We do not consider the parts of the penumbra below the red lines shown in Figs. 7a and $b$, since the field structure there is strongly disturbed by a light bridge and an arch filament system connecting this region to points outside the FOV.

The radial dependences of $B$ and $(\Delta B / \Delta \log \tau)_{0.0,-2.3}$ are displayed in Figs. $7 \mathrm{c}$ and $\mathrm{d}$. On 14 (16) November, $(\Delta B / \Delta \log \tau)_{0.0,-2.3}$ has a value of $120(100) \mathrm{G} / \log \tau$ on average in the darkest part of umbra with a maximum amplitude 

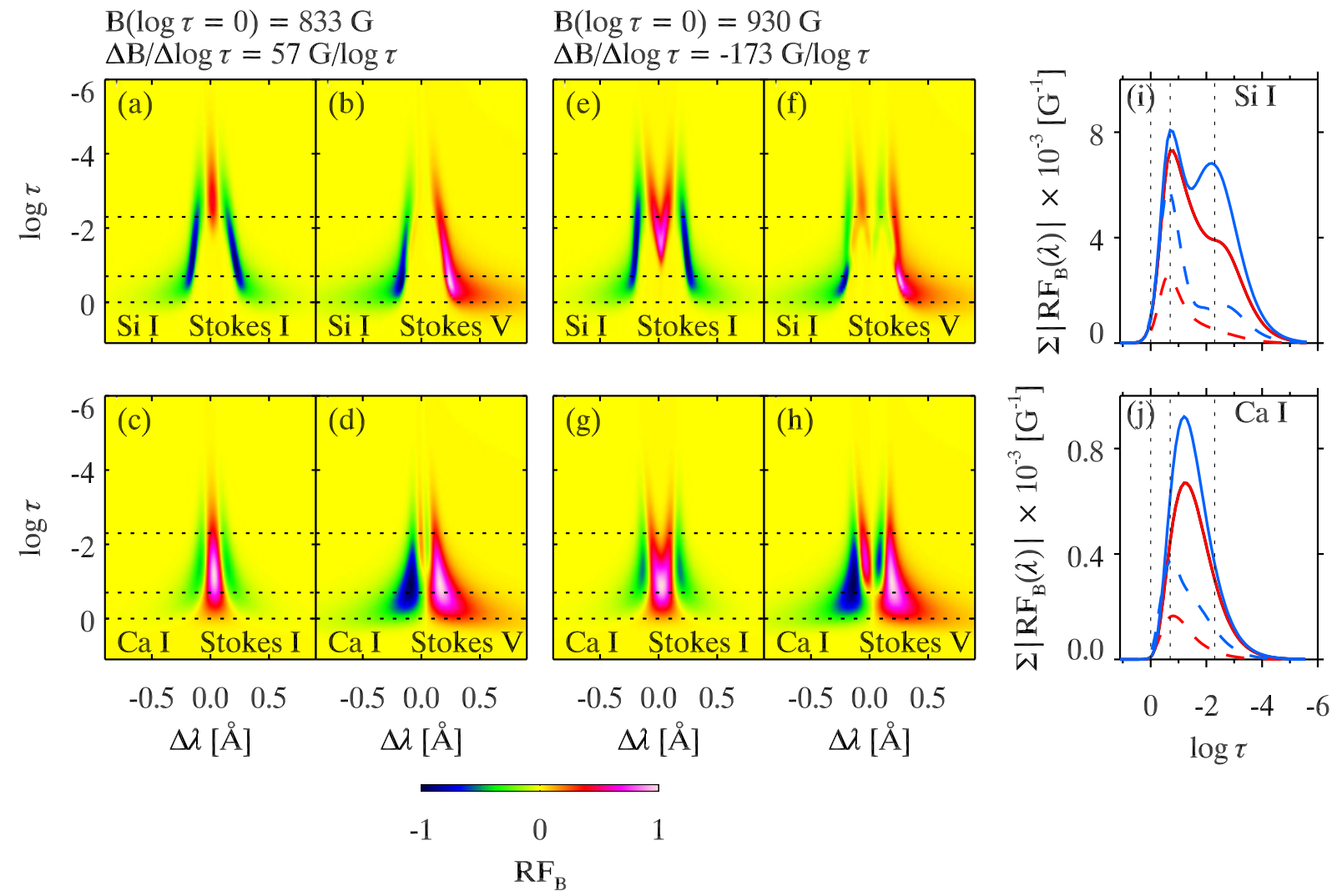

Fig. 6. Magnetic field response functions, $\mathrm{RF}_{B}$, of the Si I $10827.1 \AA$ and Ca I $10833.4 \AA$ lines. Panels a) and b) display $\mathrm{RF}_{B}$ of Stokes $I$ and Stokes $V$ of the Si I $10827.1 \AA$ line, respectively, for a penumbral atmosphere where $(\Delta B / \Delta \log \tau)$ is positive. For the same atmosphere, $R_{B}$ of $\mathrm{Ca}$ I $10833.4 \AA$ are shown in panels c) and d). Panels e)-h) are the same as a)-d), but represent $\mathrm{RF}_{B}$ of a penumbral atmosphere where $(\Delta B / \Delta \log \tau)$ is negative. Displayed $\mathrm{RF}_{B}$ are normalized to the maximum value. The absolute values of $\mathrm{RF}_{B}$ integrated along the wavelength range $\Delta \lambda= \pm 1.0 \AA$ for the Si I and Ca I lines are shown in panels i) and $\mathbf{j}$ ), respectively. The red and blue curves correspond to the positive and negative $(\Delta B / \Delta \log \tau)$, respectively. Solid curves are for Stokes $I$ and dashed curves are for Stokes $V$. Dotted horizontal or vertical lines in the panels indicate the $\log \tau$ nodes used in the inversions.

of 185 (175) G/log $\tau$. From these values we estimate that the average magnetic field gradient in the umbra on a geometrical depth scale is $\sim 1.3 \mathrm{G} \mathrm{km}^{-1}$. We converted $\Delta \log \tau$ to $\Delta d$ assuming hydrostatic equilibrium, where $d$ is the geometrical depth. In the inner penumbra, i.e., from $r / R_{\text {spot }}=0.4-0.5$ to $r / R_{\text {spot }} \simeq 0.75,(\Delta B / \Delta \log \tau)_{0.0,-2.3}$ is negative with an average value of $-40(-100) \mathrm{G} / \log \tau$. Between $r / R_{\text {spot }} \simeq 0.75$ and $r / R_{\text {spot }}=1.0$, the $(\Delta B / \Delta \log \tau)_{0.0,-2.3}$ is positive.

To cross-check the presence of negative gradients in our results we performed inversions of the Si I 10827.1 $\AA$ and Ca I $10833.4 \AA$ lines separately, but this time the atmospheric model contained a height-independent magnetic field. Comparison of the retrieved magnetic field strength from the Si I line to that from the Ca I line qualitatively confirms the vertical magnetic field gradient structure in the penumbrae obtained with the inversion of both lines together. For example, the magnetic field strength obtained from the inversion of the Ca I $10833.4 \AA$ line is $745 \mathrm{G}$ and that for the $\mathrm{Si} \mathrm{I} 10827.1 \AA$ line is $730 \mathrm{G}$. These values are for the location in the penumbra whose $\mathrm{RF}_{B}$ are shown in Figs. 6a-d. For the penumbral location corresponding to $\mathrm{RF}_{B}$ displayed in Figs. $6 \mathrm{e}-\mathrm{h}$ the retrieved magnetic field strength from the Ca I $10833.4 \AA$ and Si I $10827.1 \AA$ lines are $1190 \mathrm{G}$ and $1241 \mathrm{G}$, respectively. The average formation heights for the Ca I and $\mathrm{Si} \mathrm{I}$ lines in the penumbral atmospheres are $\log \tau=-1.6$ and $\log \tau=-2.1$, respectively. These field strength values confirm the opposite signs of the gradients obtained by inverting the two lines together.

\section{Hinode SOT/SP observations}

Low spatial resolution observations of a sunspot can lead to under- or overestimation of $B$ and $(\Delta B / \Delta \log \tau)$ due to unresolved multiple magnetic components with different orientation of the magnetic vector within the resolution element. This effect is caused by signal cancellation of Stokes $V$ profiles. The unexpected result that $B$ decreases with optical depth in azimuthal averages in the inner penumbra leads to the question: Is this outcome a spurious result of the low spatial resolution of the VTT/TIP-2 data or is it real?

To answer this question we analyze high spatial resolution Hinode SOT/SP observations. The SOT/SP records full Stokes profiles of the Fe I $6301.5 \AA$ and Fe I $6302.5 \AA$ spectral lines. The SOT/SP observations analyzed here belong to active region NOAA 10933, which were taken on 5 January 2007 close to disk center $\left(5^{\circ} \mathrm{N}, 2^{\circ} \mathrm{W}\right)$. This data set was inverted by Tiwari et al. $(2013,2015)$ and van Noort et al. (2013), and we employed the atmospheric parameters retrieved as part of these studies. These authors used the spatially coupled inversion technique of van Noort (2012) to infer atmospheric parameters. The particular inversion considered here was performed with an enhanced spatial sampling of 0.08 , compared to 0 "' 16 of the original Hinode SOT/SP (see van Noort et al. 2013, for details). The initial atmospheric model used by Tiwari et al. (2013) consists of three nodes each for $T, v_{\mathrm{los}}, B, \gamma, \phi$, and $v_{\text {mic }}$. The three nodes are at $\log \tau=0.0,-0.9$, and -2.5 . 
(a) 14.11.2010

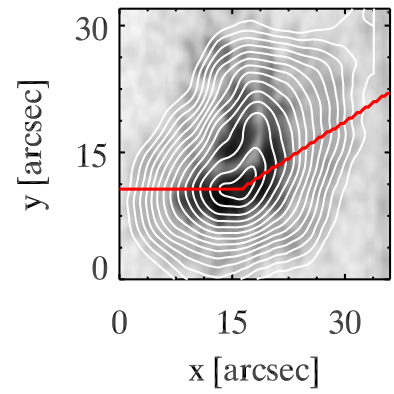

(c)

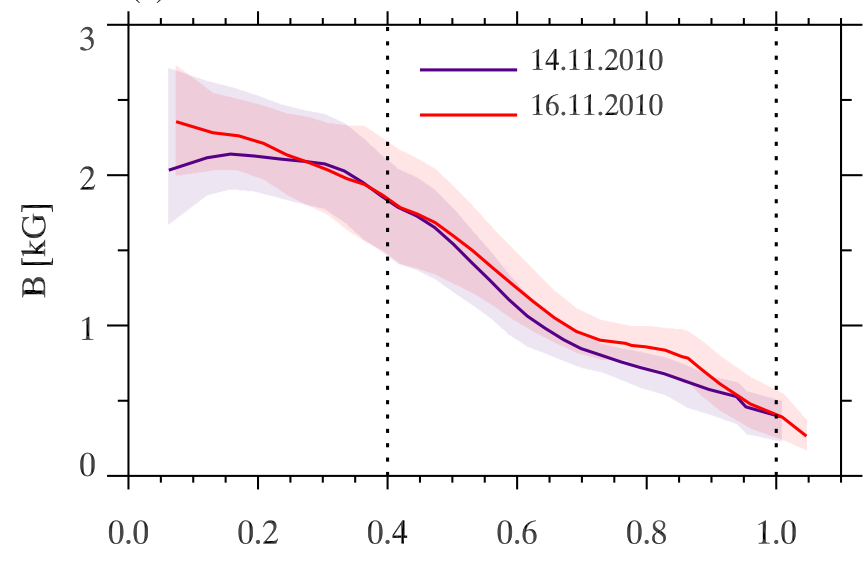

(d)

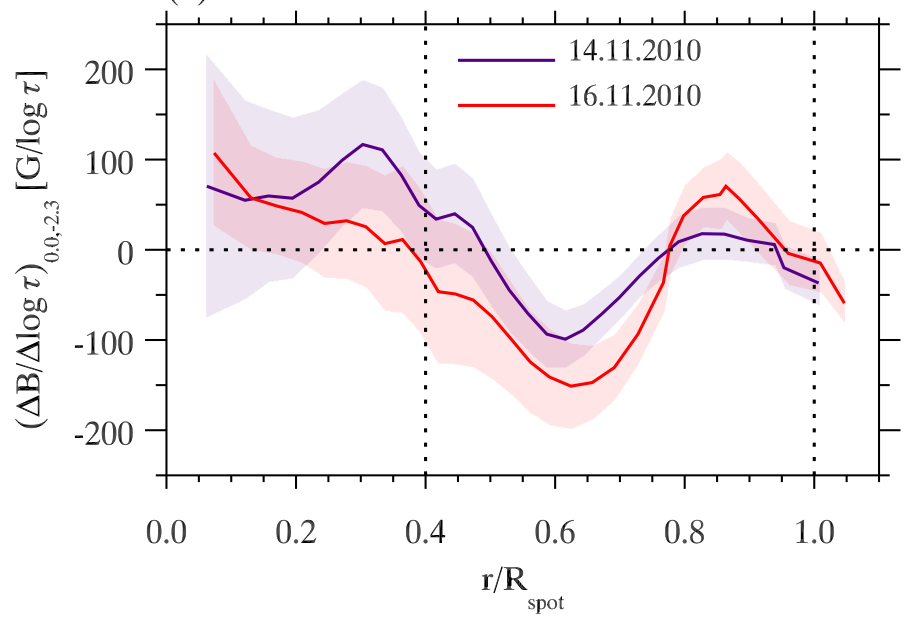

Fig. 7. Continuum intensity maps from 14 November 2010 (panel a)) and 16 November 2010 (panel b)). The contours are used to calculate azimuthal averages. The areas above the red lines were neglected because of the complex structure of the sunspot there. Azimuthally averaged parameters: Panel c) shows the radial dependence of $B$ of the sunspot observed on 14 November 2010 (violet curve) and 16 November 2010 (red curve) with VTT/TIP-2. Panel d) represents the vertical gradient of the magnetic field strength $(\Delta B / \Delta \log \tau)_{0.0,-2.3}$ as a function of $r / R_{\text {spot }}$. Shaded areas represent standard deviations. Dotted vertical lines in panels c) and d) indicate the averaged location of the umbrapenumbra boundary and the outer boundary of the sunspot.

The map of continuum intensity and $(\Delta B / \Delta \log \tau)$ maps are shown in Fig. 8. The map of $(\Delta B / \Delta \log \tau)_{0.0,-0.9}$, (panel b) shows patches of negative values in the inner penumbra, i.e., at these locations $B$ decreases with optical depth. Patches of negative values also exist in the map of $(\Delta B / \Delta \log \tau)_{-0.9,-2.5}$, i.e., in the upper atmospheric layer (see panels $\mathrm{c}$ of Fig. 8), but they are smaller in number and with lower amplitude. For simplicity we denote the atmosphere between $\log \tau=0$ and -0.9 the lower layer, and between $\log \tau=-0.9$ and -2.5 the upper layer. Just outside the visible boundary of the sunspot there is a ring of negative gradient in both the lower and upper atmospheric layers. This ring of negative gradient extends farther in the upper layer than in the lower layer.

The stratification of magnetic field strength with respect to $\log \tau$ at two different locations in the penumbra are shown in Fig. 9. Panels $\mathrm{b}$ and $\mathrm{c}$ display $\mathrm{RF}_{B}$ of Stokes $I$ and $V$ profiles of the Fe I $6301.5 \AA$ and Fe I $6302.5 \AA$ lines for the atmosphere in panel a where the magnetic field strength increases with optical depth. Panels e and $f$ represent those for the atmosphere depicted in panel $\mathrm{d}$ where the magnetic field strength decreases with optical depth. The $\mathrm{RF}_{B}$ for these atmospheres demonstrate that $\log \tau$ nodes used for the inversions of the Fe I $6301.5 \AA$ and Fe I $6302.5 \AA$ lines reasonably samples their formation heights.

The gradient maps are highly structured; this can be seen in Fig. 10, which is a zoomed-in version of Fig. 8 with magnetic field inclination information added. Contours in panels a and $\mathrm{c}-\mathrm{f}$ of Fig. 10 separate patches with negative and positive values of $(\Delta B / \Delta \log \tau)_{0.0,-0.9}$. It is evident that the patches where the field has a negative gradient mostly coincide with bright filaments. The gradient is steeper in the lower layer (panel b) than in the upper layer (panel c). The gradient also shows fine structure within the body of individual filaments. Most parts of the filaments display negative values of $(\Delta B / \Delta \log \tau)_{0.0,-0.9}$, while at the centers of the filaments $(\Delta B / \Delta \log \tau)_{0.0,-0.9}$ is positive (indicated by an arrow in panel b of Fig. 10). In the upper layer the filaments have mostly negative $(\Delta B / \Delta \log \tau)_{-0.9,-2.5}$ along their central parts, in particular near their heads. Contours colored in white in panel $\mathrm{b}$ of Fig. 10 show locations where the polarity of $B$ is opposite to that of the umbra at $\log \tau=0.0$. Narrow patches of opposite polarity at $\log \tau=0.0$ are co-located with the patches of negative $(\Delta B / \Delta \log \tau)_{0.0,-0.9}$, but the former are narrower. Opposite polarity patches disappear at $\log \tau=-2.5$, whereas at $\log \tau=-0.9$ opposite polarity patches are located only at the tails of filaments.

Most of the filaments in our data set display the properties mentioned above. To check whether they are generic, we consider the averaged penumbral filament constructed by Tiwari et al. (2013), who averaged 60 penumbral filaments, 20 each from the inner, middle, and outer penumbra. Prior to averaging they straightened all filaments and then normalized their lengths. The inner, middle, and outer filaments exhibit strong similarities in all physical properties, such as the plasma flow and the magnetic field. The temperature structure is also similar, although it shows some changes with the radial distance from the sunspot center. Some of the physical properties of this averaged filament are displayed in Fig. 11. The structure of the gradient of the averaged filament is similar to that noticed in individual filaments (see Fig. 10), although much smoother. At the center along the central ridge of the filament, $(\Delta B / \Delta \log \tau)_{-0.0,-0.9}$ is positive, surrounded by negative $(\Delta B / \Delta \log \tau)_{-0.0,-0.9}$ at the edges, in particular around the head. The inner half (i.e., near the umbra) $(x<0.5$ in Fig. 11) of the filament has negative $(\Delta B / \Delta \log \tau)_{-0.9,-2.5}$ along its central ridge. The outer half $(x>0.5)$ displays positive values of $(\Delta B / \Delta \log \tau)_{-0.9,-2.5}$.

To determine the penumbral magnetic field structure that can produce such a magnetic field gradient, it is important to see how the magnetic field is stratified with optical depth. The optical depth stratification of various physical parameters is shown in Fig. 12. As mentioned before, the atmosphere is fitted only on the three $\log \tau$ positions. The stratification at intermediate $\log \tau$ 


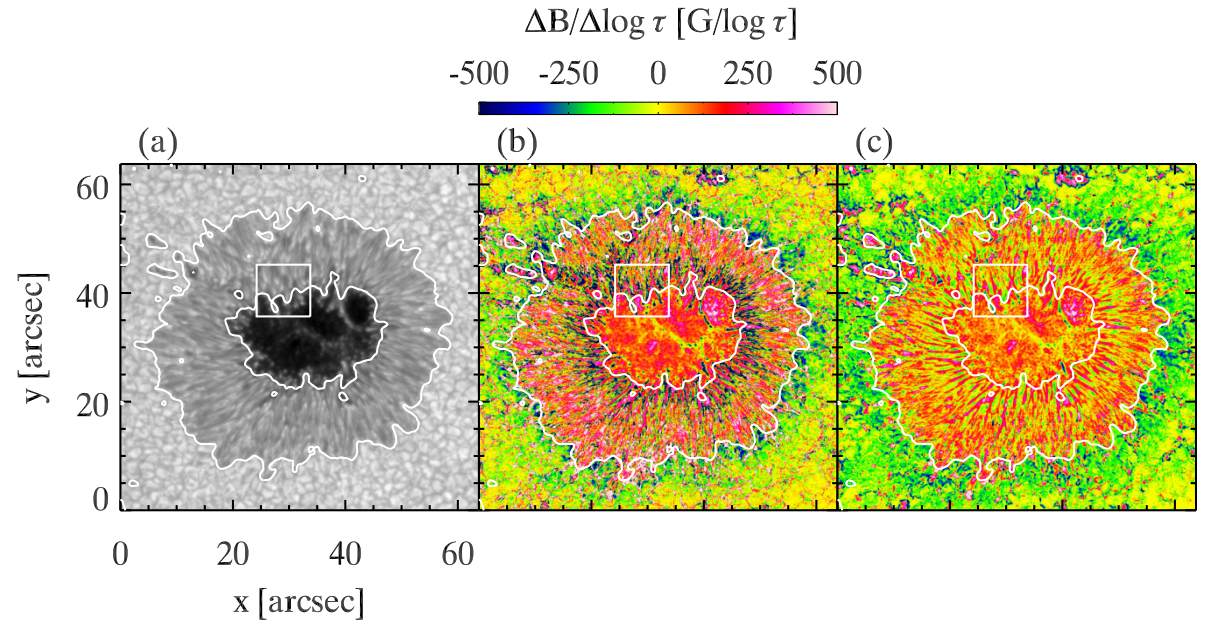

Fig. 8. Continuum intensity (panel a)) and $(\Delta B / \Delta \log \tau)$ maps of a sunspot in active region NOAA 10933, observed with Hinode SOT/SP. Panels b) and c) represent $(\Delta B / \Delta \log \tau)_{0.0,-0.9}$ and $(\Delta B / \Delta \log \tau)_{-0.9,-2.5}$, respectively. Inner and outer white contours in all panels represent the umbra-penumbra boundary and the outer boundary of the sunspot, respectively.
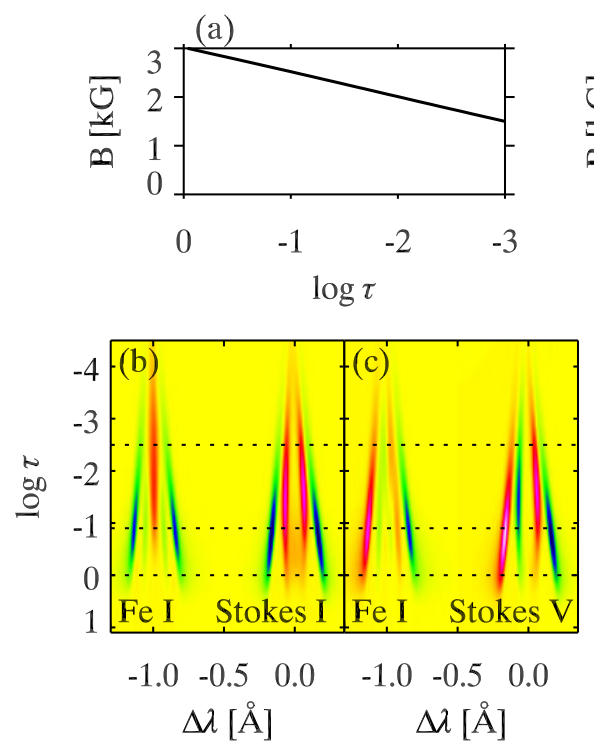
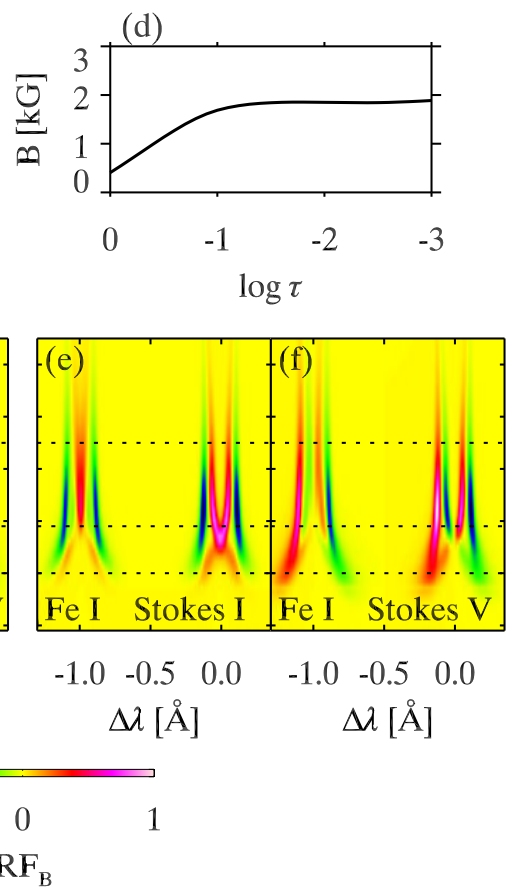

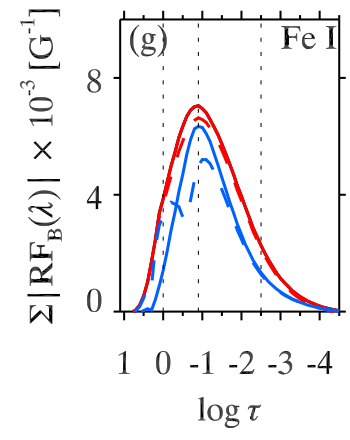

$\begin{array}{ccc}-1 & 0 & 1 \\ & \mathrm{RF}_{\mathrm{B}} & \end{array}$

Fig. 9. Magnetic field response functions, $\mathrm{RF}_{B}$, of the Fe I $6301.5 \AA$ and Fe I $6302.5 \AA$ lines. Panels b) and c) show RF ${ }_{B}$ for Stokes $I$ and Stokes $V$, respectively, for a penumbral atmosphere with the magnetic field strength stratification presented in panel a). Panels e) and f) are the same as panels b) and c) but correspond to the magnetic field stratification displayed in panel d). The presented $\mathrm{RF}_{B}$ values are normalized to the maximum value. The absolute values of $\mathrm{RF}_{B}$, integrated over wavelength $(\Delta \lambda= \pm 0.35 \AA)$ for the Fe $\mathrm{I} 6302.5 \AA$ line, are shown in panel g). Red and blue curves correspond to the positive and negative $(\Delta B / \Delta \log \tau)$, respectively. Solid curves are for Stokes $I$ and dashed curves are for Stokes $V$. Dotted horizontal lines in panels b); c); e); and f) and dotted vertical lines in panel $\mathbf{g}$ ) indicate the log $\tau$ nodes used in the inversions.

positions shown in Fig. 12 is derived by interpolation using the same bicubic spline approximation as was used during the inversion procedure. The positions of these vertical cuts through the standard filament displayed in Fig. 12 are indicated by dashed vertical lines in Fig. 11. In the central ridge $\left(\Delta y=0^{\prime \prime}\right)$ of the head of the filament (first column of Fig. 12), $B$ becomes weaker and more horizontal from $\log \tau=0.0$ to $\log \tau=-0.9$ and then increases again from $\log \tau=-0.9$ to $\log \tau=-2.5$, so that the vertical gradient of the field is positive between $\log \tau=0.0$ and $\log \tau=-0.9$ and negative between $\log \tau=-0.9$ and $\log \tau=-2.5$. Between $\log \tau=0.0$ and $\log \tau=-0.9$ the vertical component of the magnetic field, $B_{\mathrm{z}}$, also becomes weaker, whereas the radial component of the magnetic field, $B_{r}$, becomes stronger. At the edge of the filament head $(\Delta y \simeq \pm 0$ "' 4$), B$ is horizontal and weaker in the deeper atmosphere and becomes more vertical and stronger in the upper atmosphere. The more vertical and stronger field in the upper part of the atmosphere is thought to be a field from the surrounding spines, expanding and closing above the filament, which carries weaker magnetic fields. This might explain why we see a negative field gradient at the location of the filament.

At the middle and tail part of the filament (second and third columns of Fig. 12) the horizontal field at the filament center is stronger than the overlying more vertical field. Hence in the middle and tail part of the bright filament the field gradient is positive.

To derive the dependence of $(\Delta B / \Delta \log \tau)$ on the radial distance from the sunspot center we use the same method as applied 
$\Delta \mathrm{B} / \Delta \log \tau[\mathrm{G} / \log \tau]$

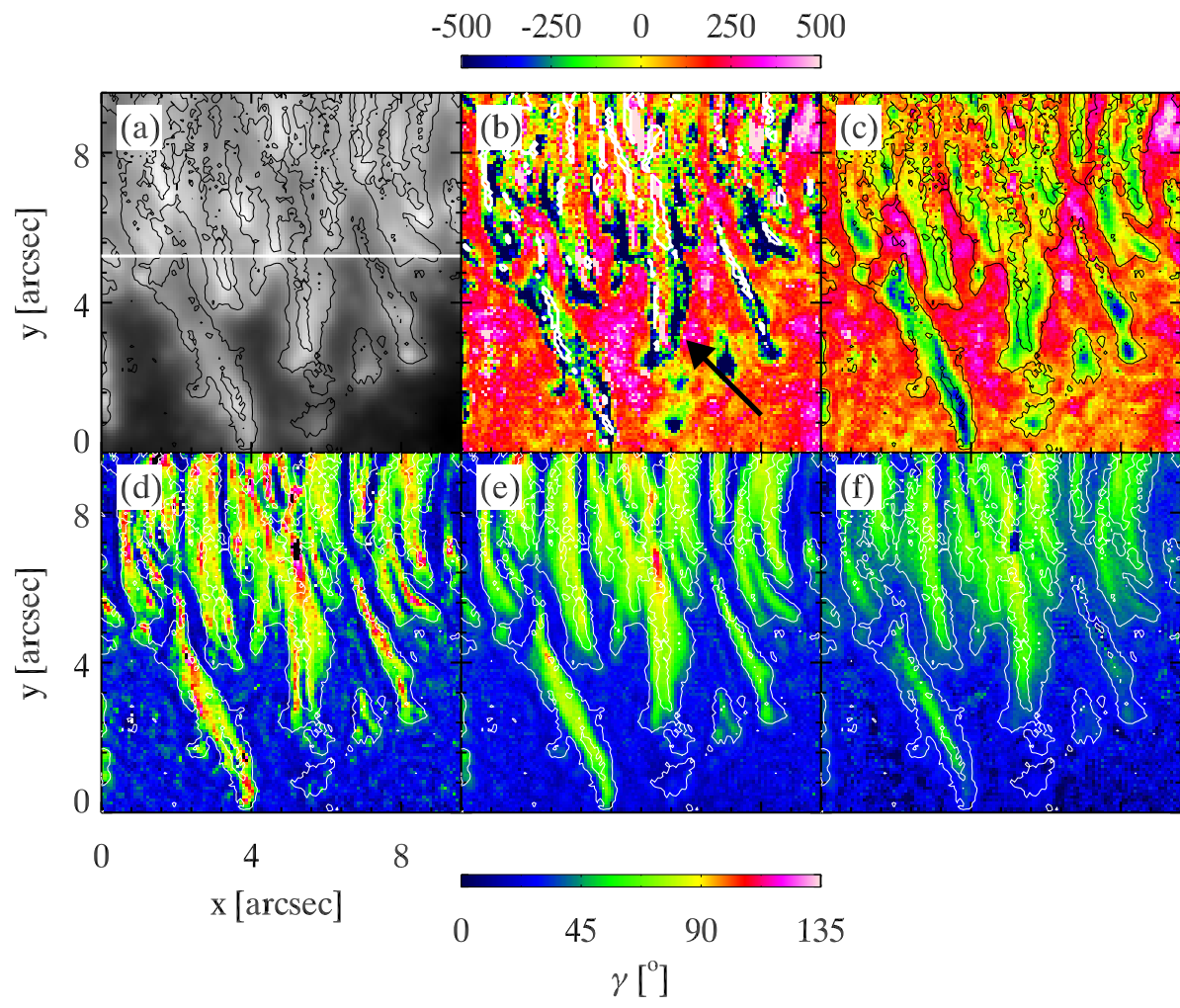

Fig. 10. a)-c): Same as Fig. 8, but showing only the area within the white box in panel a) of Fig. 8. Additional panels d), e), and f) display the inclination of the magnetic field at $\log \tau=0.0,-0.9,-2.5$, respectively. Contours in panels a); c); d); e); and f) separate patches of negative and positive $(\Delta B / \Delta \log \tau)_{0.0,-0.9}$ and white contours in panel $\mathbf{b})$ indicate areas of polarity opposite to that in the umbra at $\log \tau=$ 0.0 . The horizontal white line in panel a) indicates the location of the cut plotted in Fig. 14. The black arrow in panel b) marks an example of a penumbral filament with negative $(\Delta B / \Delta \log \tau)_{0.0,-0.9}$ at its edges.

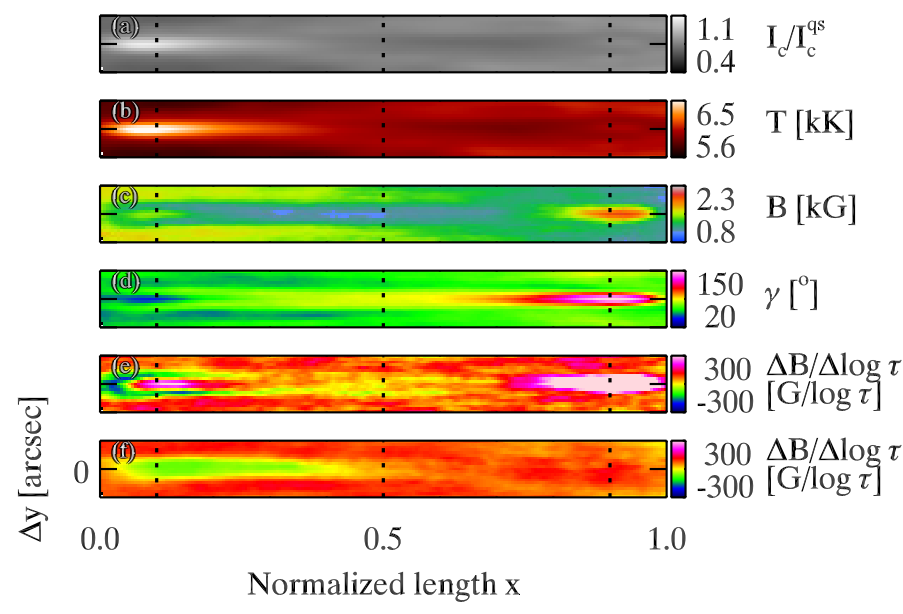

Fig. 11. Physical properties of the standard filament from Tiwari et al. (2013). a) Continuum intensity normalized to the quiet Sun continuum; b) $T$ at $\log \tau=0.0$; c) $B$ at $\log \tau=0.0$; d) $\gamma$ at $\log \tau=0.0$; e) $(\Delta B / \Delta \log \tau)_{0.0,-0.9}$; and f) $(\Delta B / \Delta \log \tau)_{-0.9,-2.5}$. The left- and rightmost part of the panels correspond to the inner and outer part of the filament, respectively, with inner meaning closer to the umbra.

to the VTT/TIP-2 data (see Sect. 2.3). The radial dependence of $(\Delta B / \Delta \log \tau)_{0.0,-0.9}$ shows a qualitative similarity with that of $(\Delta B / \Delta \log \tau)_{0.0,-2.3}$ found from the VTT/TIP-2 observations, but the gradients are more positive. Quantitatively, the positive values retrieved from the SOT/SP observations are higher: in the umbra the average value of $(\Delta B / \Delta \log \tau)_{0.0,-0.9}$ is found to be $150 \mathrm{G} / \log \tau$ (see Fig. 13), $\sim 1.4 \mathrm{G} \mathrm{km}^{-1}$ in the geometrical depth scale. In the inner penumbra, between $r \simeq 0.43 R_{\text {spot }}$ and $r \simeq 0.63 R_{\text {spot }},(\Delta B / \Delta \log \tau)_{0.0,-0.9}$ is negative with the maximum negative value of $-80 \mathrm{G} / \log \tau$ being reached at $r=0.5 R_{\text {spot }}$.
The sign of $(\Delta B / \Delta \log \tau)_{0.0,-0.9}$ is positive beyond $r \simeq 0.6 R_{\text {spot }}$; $(\Delta B / \Delta \log \tau)_{-0.9,-2.5}$ amounts to $120 \mathrm{G} / \log \tau$ on average in the umbra and also remains positive in the penumbra. Outside the boundary of the sunspot, on average $(\Delta B / \Delta \log \tau)_{-0.9,-2.5}$ is negative.

Figure 14 displays $(\Delta B / \Delta \log \tau)_{0.0,-0.9}$ and $I_{\mathrm{c}} / I_{\mathrm{c}}^{\mathrm{qs}}$ along a slit (corresponding to the horizontal white line in Fig. 10a) in the penumbra cutting across several penumbral filaments. The parameters $I_{\mathrm{c}}$ and $I_{\mathrm{c}}^{\mathrm{qs}}$ represent continuum intensity and averaged continuum intensity in the quiet Sun, respectively. Along the slit both positive and negative patches of $(\Delta B / \Delta \log \tau)_{0.0,-0.9}$ are present. At the location of the filaments, $(\Delta B / \Delta \log \tau)_{0.0,-0.9}$ has negative values with larger magnitudes than the positive $(\Delta B / \Delta \log \tau)_{0.0,-0.9}$ of the dark background. Hence, the azimuthal average of $(\Delta B / \Delta \log \tau)_{0.0,-0.9}$ turns out to be negative in the inner penumbra.

\section{Comparison with 3D MHD simulations}

To better understand the origin of the azimuthally averaged negative $(\Delta B / \Delta \log \tau)_{0.0,-0.9}$ and $(\Delta B / \Delta \log \tau)_{0.0,-2.3}$ in sunspot penumbrae detected in the SOT/SP and VTT/TIP-2 observations, respectively, we analyzed a snapshot from a 3D MHD simulation of a sunspot by Rempel (2012) (cf. Rempel 2011). The advantage of studying the MHD simulation is that we can also determine the gradient on a geometrical depth scale, $d$, rather than just on an optical depth scale.

The azimuthally averaged $B$ and $\partial B / \partial d$ stratified with respect to geometrical depth are depicted in Fig. 15a and b, respectively. In Figs. 15, $d$ denotes the geometrical depth ( $d$ increases into the Sun) and $d_{\text {photo }}$ is the average geometrical depth in the quiet Sun photosphere at $\log \tau=0$. The considerable difference 

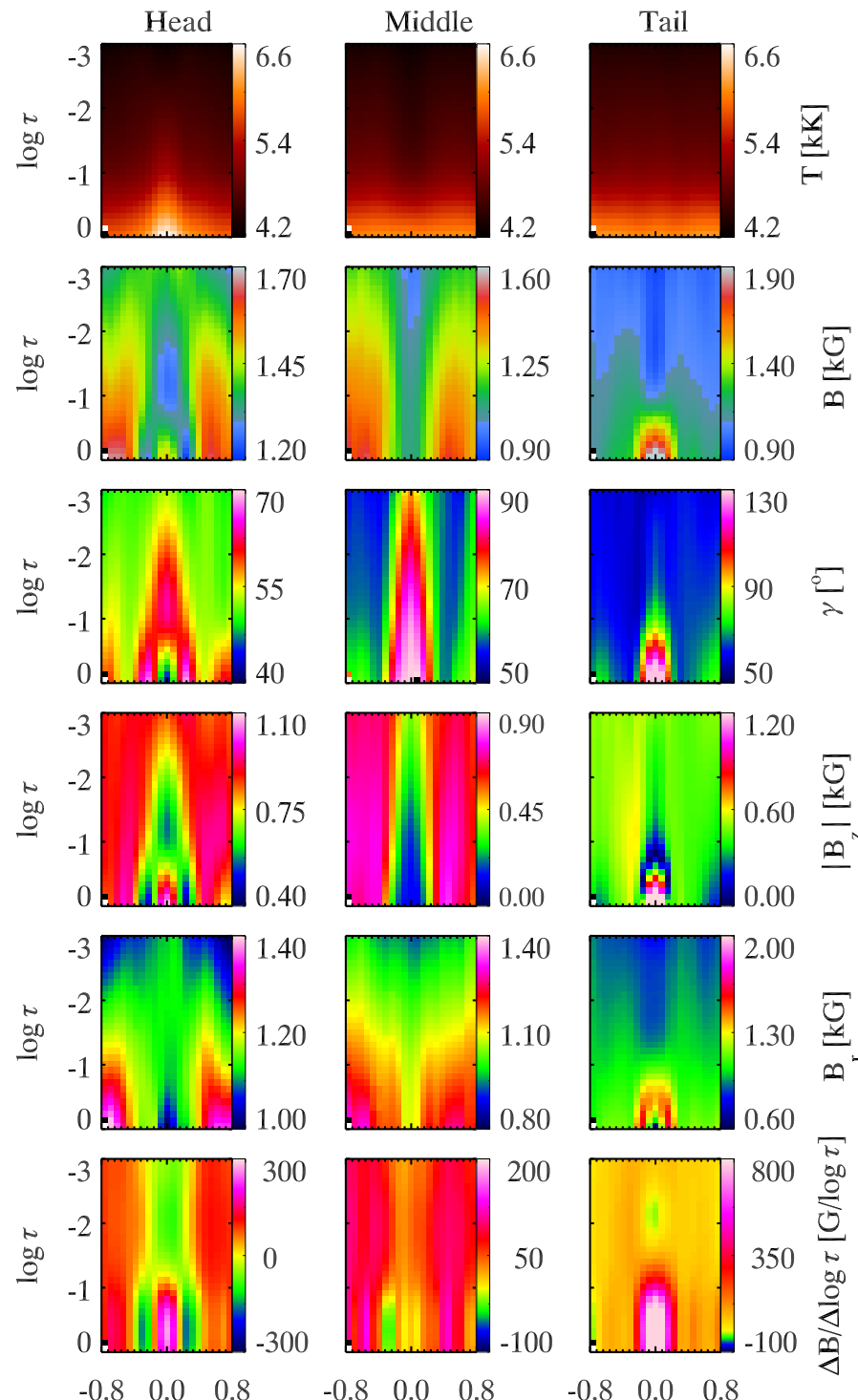

$-0.8 \quad 0.0 \quad 0.8$

$\Delta y[\operatorname{arcsec}]$
$\Delta y[\operatorname{arcsec}]$

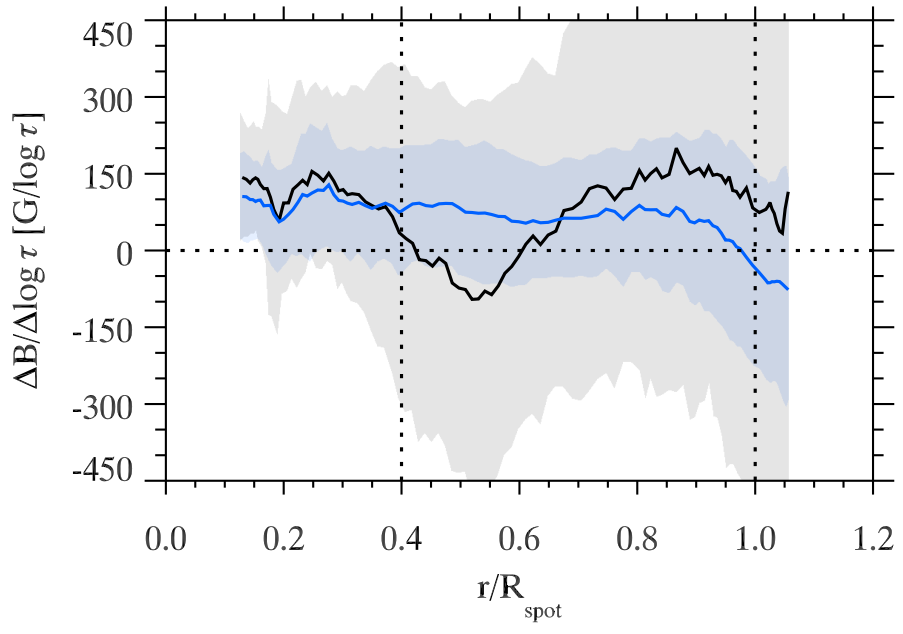

Fig. 13. Azimuthally averaged magnetic field gradient as a function of normalized radial distance, $r / R_{\text {spot }}$, from the center of the sunspot observed with SOT/SP. Black and blue curves depict $(\Delta B / \Delta \log \tau)_{0.0,-0.9}$ and $(\Delta B / \Delta \log \tau)_{-0.9,-2.5}$, respectively. Shaded areas represent the standard deviations. Dotted vertical lines indicate the umbra-penumbra boundary and the outer boundary of the sunspot.

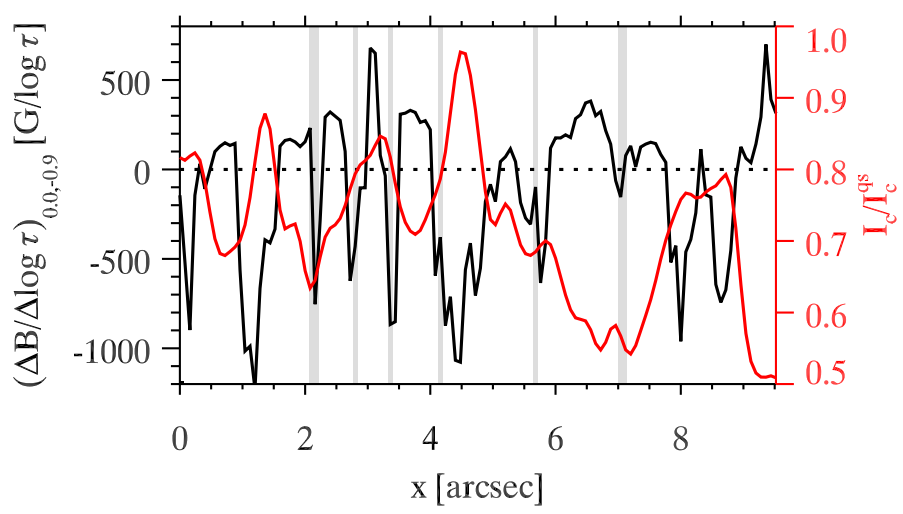

Fig. 14. Fluctuations of $(\Delta B / \Delta \log \tau)_{0.0,-0.9}$ (black curve) and $I_{\mathrm{c}} / I_{\mathrm{c}}^{\mathrm{qs}}$ (red curve) along the white line in Fig 10a. Gray shaded areas correspond to positions with polarity opposite to that in the umbra.

simulation (see dashed horizontal lines in Fig. 15a and b). The value of $(\Delta B / \Delta d)$ is defined as follows,

$\left(\frac{\Delta B}{\Delta d}\right)_{\mathrm{p}, \mathrm{q}}=\frac{B(p)-B(q)}{p-q}$,

where $p$ and $q$ denotes the lower and upper geometrical depth, $d-d_{\text {photo }}$, respectively.

Azimuthal averages of $(\Delta B / \Delta d)$ calculated for three depth intervals are presented in Fig. 15c. The $\log \tau=0$ surface in the inner penumbra in the MHD simulation is on average $\sim 400 \mathrm{~km}$ deeper than in the quiet Sun, so we do not look at gradients of $B$ below $d-d_{\text {photo }}=384 \mathrm{~km}$. It shows an absence of negative $(\Delta B / \Delta d)_{192,0}$ and $(\Delta B / \Delta d)_{0,-192}$ within the sunspot although these gradients achieve negative values outside the boundary of the sunspot. The gradient in the lowest considered layer, $(\Delta B / \Delta d)_{384,192}$, exhibits negative values for $0.9 \gtrsim r / R_{\text {spot }} \gtrsim 0.6$. The shaded areas in Fig. $15 \mathrm{c}$ show standard deviations of $(\Delta B / \Delta d)$ along the circles used to estimate the azimuthal averages.

Figure 16 shows a continuum intensity map at $6303.1 \AA$ (panel a) and maps of $(\Delta B / \Delta d)_{384,192},(\Delta B / \Delta d)_{192,0}$, and $(\Delta B / \Delta d)_{0,-192}$ in panels a-d, respectively. There are patches netic field between given geometrical depths from the MHD 


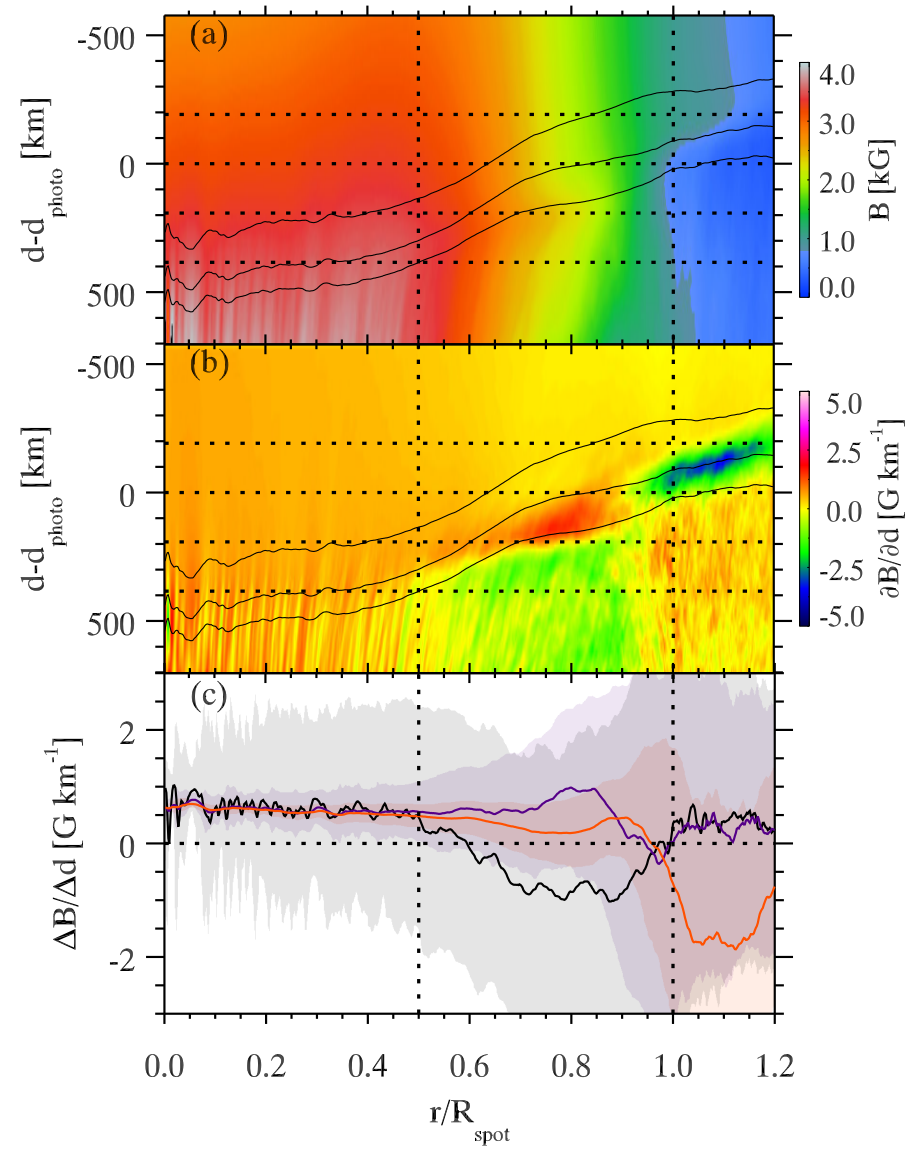

Fig. 15. Azimuthally averaged magnetic field and its vertical gradient in the 3D MHD simulation. Panel a) represents the magnetic field as a function of normalized radial distance $r / R_{\text {spot }}$ and geometrical depth $d-d_{\text {photo }}$, where $d_{\text {photo }}$ denotes the average geometrical depth of the quiet Sun photosphere at $\log \tau=0$. Panel b) displays $\partial B / \partial d$. The lower, middle, and upper curves in panels a) and b) show the $\log \tau=$ $0.0, \log \tau=-0.9$, and $\log \tau=-2.5$ levels, respectively. The black, blue, and red curves in panel c) depict $(\Delta B / \Delta d)$ estimated between $d-d_{\text {photo }}=384 \mathrm{~km}$ and $192 \mathrm{~km}, d-d_{\text {photo }}=192 \mathrm{~km}$ and $0 \mathrm{~km}$, and $d-d_{\text {photo }}=0 \mathrm{~km}$ and $-192 \mathrm{~km}$, respectively. These geometric depth positions used in estimating $(\Delta B / \Delta d)$ are shown by horizontal lines in panels a) and b). Shaded areas represent standard deviations. Dotted vertical lines in all panels indicate the umbra-penumbra boundary and the outer boundary of the sunspot.

where $(\Delta B / \Delta d)_{384,192}$ is negative for $0.9 \gtrsim r / R_{\text {spot }} \gtrsim 0.6$. We see fewer patches in the penumbra where $(\Delta B / \Delta d)_{192,0}$ or $(\Delta B / \Delta d)_{0,-192}$ is negative compared to $(\Delta B / \Delta \log \tau)_{0.0,-0.9}$ in the Hinode SOT/SP and VTT/TIP-2 observations.

As mentioned above, the azimuthally averaged $(\Delta B / \Delta d)_{192,0}$ and $(\Delta B / \Delta d)_{0,-192}$ in the MHD simulation does not display negative gradients in the inner penumbra, whereas the SOT/SP and VTT/TIP-2 observations show that the vertical gradient (in optical depth scales) of the inner penumbra is negative in the azimuthal averages. There are several possible reasons for this discrepancy between the MHD simulation and the observations:

1. The gradients of $B$ related to geometrical depth may not be directly comparable to gradients at a given optical depth (since iso-optical depth surfaces are likely strongly corrugated in the azimuthal direction, depending on the field strength and the temperature). Also, a given optical depth samples different geometrical depths at various radii in the penumbra.
2. There are shortcomings in the simulations that lead to a departure from realism in the synthetic sunspot's magnetic structure. For example, the penumbra is rather shallow in the MHD simulation and the lowest layer analyzed here $\left(d-d_{\text {photo }}=384 \mathrm{~km}\right)$ is below the lower boundary of the magnetic field at some locations at $r / R_{\text {spot }} \gtrsim 0.6$. This may explain some of the negative $(\Delta B / \Delta d)_{384,192}$ at larger $r / R_{\text {spot }}$ in the simulation.

3. The inversions of the observed Stokes profiles may be imperfect regarding the magnetic structure, although the inversions of the two rather diverse observed data sets (different spectral lines, different spatial resolution, ground-based vs. space-borne, etc.) analyzed using contrasting approaches (stray light and PSF removed vs. non-consideration of stray light) result in qualitatively similar magnetic field gradients.

For a comparison of the MHD atmosphere with the VTT/TIP2 and SOT/SP observations according to point (1), we computed $(\Delta B / \Delta \log \tau)$ between two iso- $\tau$ surfaces, determined using the SPINOR code, everywhere in the simulated sunspot following Eq. (2). The resulting maps are shown in Fig. 17. The $(\Delta B / \Delta \log \tau)_{0.0,-0.9}$ map reveals patches with negative values between $r / R_{\text {spot }} \approx 0.4$ and 0.7 , similar to what is seen in the SOT/SP observations. Patches with negative values in the $(\Delta B / \Delta \log \tau)_{-0.9,-2.5}$ and $(\Delta B / \Delta \log \tau)_{-2.5,-3.5}$ maps are, however, shifted to larger radii, lying between $r / R_{\text {spot }} \approx 0.7$ and $r / R_{\text {spot }} \approx$ 1.0. This behavior is not visible in the SOT/SP data. There the patches with negative values of $(\Delta B / \Delta \log \tau)$ are mostly found in the inner penumbra, also for the upper layer (see Fig. 8). At the boundary of the sunspot we see a ring where $(\Delta B / \Delta \log \tau)_{0.0,-0.9}$ is negative. A much more prominent ring is located farther out in $(\Delta B / \Delta \log \tau)_{-0.9,-2.5}$ map. This is likely associated with the magnetic canopy surrounding the sunspot.

Blowups of the region inside the white squares in Fig. 17 are displayed in Fig. 18. In the MHD simulation too, patches where $(\Delta B / \Delta \log \tau)_{0.0,-0.9}$ is negative coincide with bright filaments. In the lower layers, patches with negative $(\Delta B / \Delta \log \tau)$ exist only around the heads of the filaments. In the upper layers large parts of the filament's body show a weak negative $(\Delta B / \Delta \log \tau)$. It is important to note that there is no clear transition from peripheral umbral dots to penumbral filaments in the inner penumbra (defined according to azimuthally averaged intensity) of the MHD simulations. The penumbral filaments discussed here lies between $r / R_{\text {spot }} \simeq 0.4$ and $r / R_{\text {spot }} \simeq 0.75$, and regarding some physical properties (for example plasma flow) they are may be more similar to peripheral umbral dots than penumbral filaments. Although, the described properties of the vertical magnetic field gradients are nearly valid for the penumbral filaments in the outer penumbra.

The azimuthally averaged $(\Delta B / \Delta \log \tau)$ values as derived from the MHD simulation, again as a function of the normalized sunspot radius, are shown in Fig. 19. The shaded areas represent standard deviations. The radial dependence of $(\Delta B / \Delta \log \tau)_{0.0,-0.9}$ is qualitatively similar to that of the SOT/SP observations showing negative values $(-25 \mathrm{G} / \log \tau)$ in the inner penumbra. However, these negative gradients are much weaker than in the observations. In the middle and outer penumbra $(\Delta B / \Delta \log \tau)_{0.0,-0.9}$ is positive but with some quantitative differences to the SOT/SP observations. The azimuthally averaged $(\Delta B / \Delta \log \tau)_{-0.9,-2.5}$ in the MHD simulation is positive at all radial distances within the sunspot, which is similar to that of the SOT/SP observations. Outside the sunspot $(\Delta B / \Delta \log \tau)_{-0.9,-2.5}$ is negative. The standard deviations of the fluctuations of $B$ in the azimuthal averages have higher values in the MHD simulation 


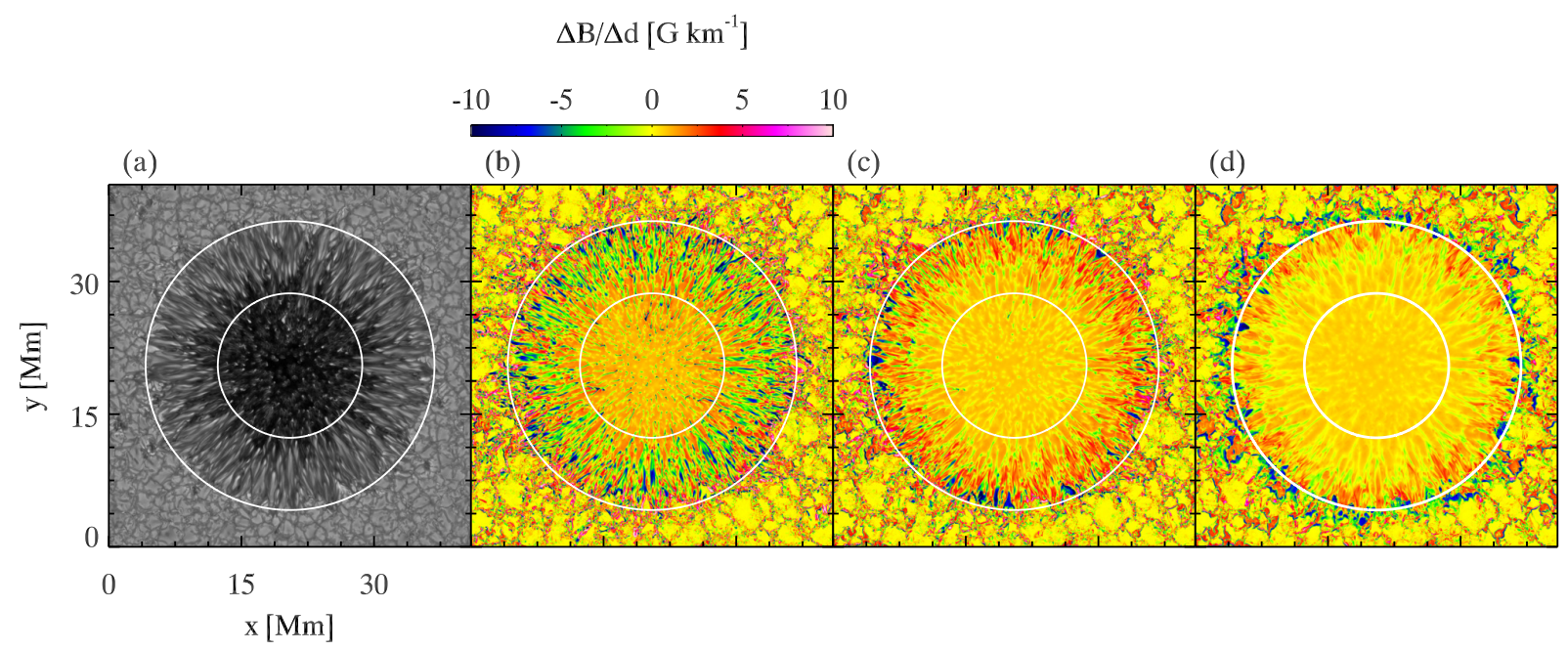

Fig. 16. Continuum intensity (panel a) and the magnetic field gradient maps from a 3D MHD simulation of a sunspot computed by Rempel (2012). Panels b-d) depict $(\Delta B / \Delta d)$ estimated between $d-d_{\text {photo }}=384 \mathrm{~km}$ and $192 \mathrm{~km}, d-d_{\text {photo }}=192 \mathrm{~km}$ and $0 \mathrm{~km}$, and $d-d_{\text {photo }}=0 \mathrm{~km}$ and $-192 \mathrm{~km}$, respectively. Here $d_{\text {photo }}$ signifies the depth at which on average $\tau=0$ is reached in the quiet Sun. White circles in all panels represent the averaged umbra-penumbra boundary and the outer boundary of the sunspot.

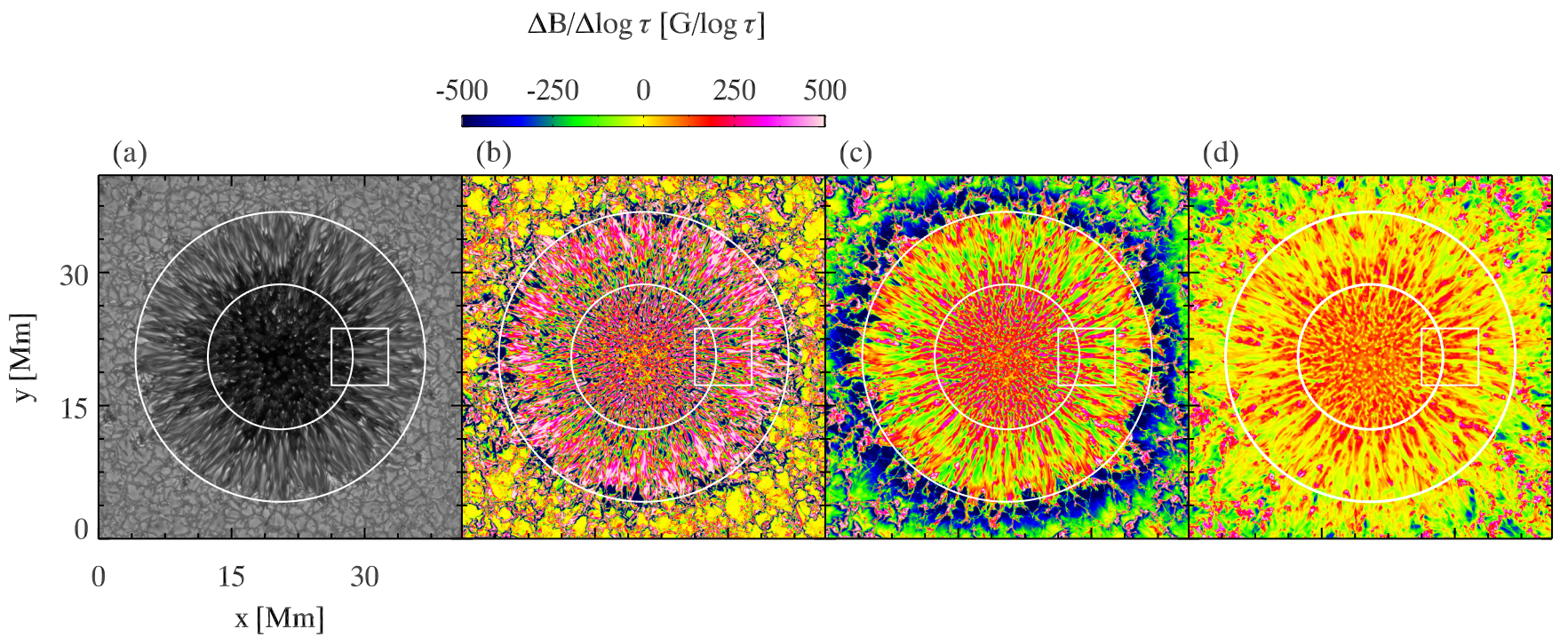

Fig. 17. Continuum intensity (panel a)) and $(\Delta B / \Delta \log \tau)$ maps obtained from the MHD simulation of a sunspot. Panels b)-d) display $(\Delta B / \Delta \log \tau)_{0.0,-0.9},(\Delta B / \Delta \log \tau)_{-0.9,-2.5}$, and $(\Delta B / \Delta \log \tau)_{-2.5,-3.5}$, respectively. The white circles have the same meaning as in Fig. 8. The white box denotes the location of the blowup displayed in Fig. 18.

compared to the SOT/SP observations. We attribute this to the higher spatial resolution of the MHD data. The azimuthal averages of the vertical gradient of the magnetic field obtained from VTT/TIP-2, SOT/SP, and the MHD simulation are compared in Table 2. The values listed in the table are averaged over the respective areas.

The azimuthally averaged $(\Delta B / \Delta \log \tau)_{0.0,-0.9}$ in the MHD simulation shows some negative values in the inner penumbra, unlike the azimuthally averaged $\partial B / \partial d$ which only has positive values above $\log \tau=0$ in the inner penumbra (see Fig. 15b). Also, the spatially resolved maps of the vertical gradient of the magnetic field have negative values patches in the geometrical and in optical depth scale, which predominantly coincide with the inner part (head) of the penumbral filaments. This suggests that the corrugation of the optical depth surfaces may play some role in producing the negative $(\Delta B / \Delta \log \tau)_{0.0,-0.9}$ in the azimuthal averages in the inner penumbra.

To investigate the effect of the corrugation of the optical depth surfaces on the azimuthally averaged $(\Delta B / \Delta \log \tau)_{0.0,-0.9}$, the height stratifications of the physical properties at the head, middle, and tail of a penumbral filament from the MHD simulation are plotted in Fig. 20. At the head of the filament $\partial B / \partial d$ has negative values between $\log \tau=0.0$ (lower white curve) and $\log \tau=-0.9$ (middle white curve), whereas it is positive in the surroundings (spines) of the filaments. The negative $\partial B / \partial d$ at the head of the filaments is caused by the closing of the stronger vertical fields from the spines above the weaker and relatively horizontal fields of the filament (inter-spines). The negative $\partial B / \partial d$ above $\log \tau=0.0$ is more prominent at the head of the filament than in the middle and tail of the filament. At the head of the filament, the extension of strong negative $\partial B / \partial d$ with geometrical depth follows the corrugation of $\log \tau=0.0$ and $\log \tau=-0.9$ levels. So in this case, areas with negative gradients (filaments) contribute more to the azimuthal averages of the vertical gradient of the magnetic field (in the geometrical depth scale) than areas with positive gradients (spines). Since there are more filament heads in the inner penumbra than in the middle and outer penumbra, the azimuthally averaged $(\Delta B / \Delta \log \tau)_{0.0,-0.9}$ is negative in 


\section{$\Delta \mathrm{B} / \Delta \log \tau[\mathrm{G} / \log \tau]$}

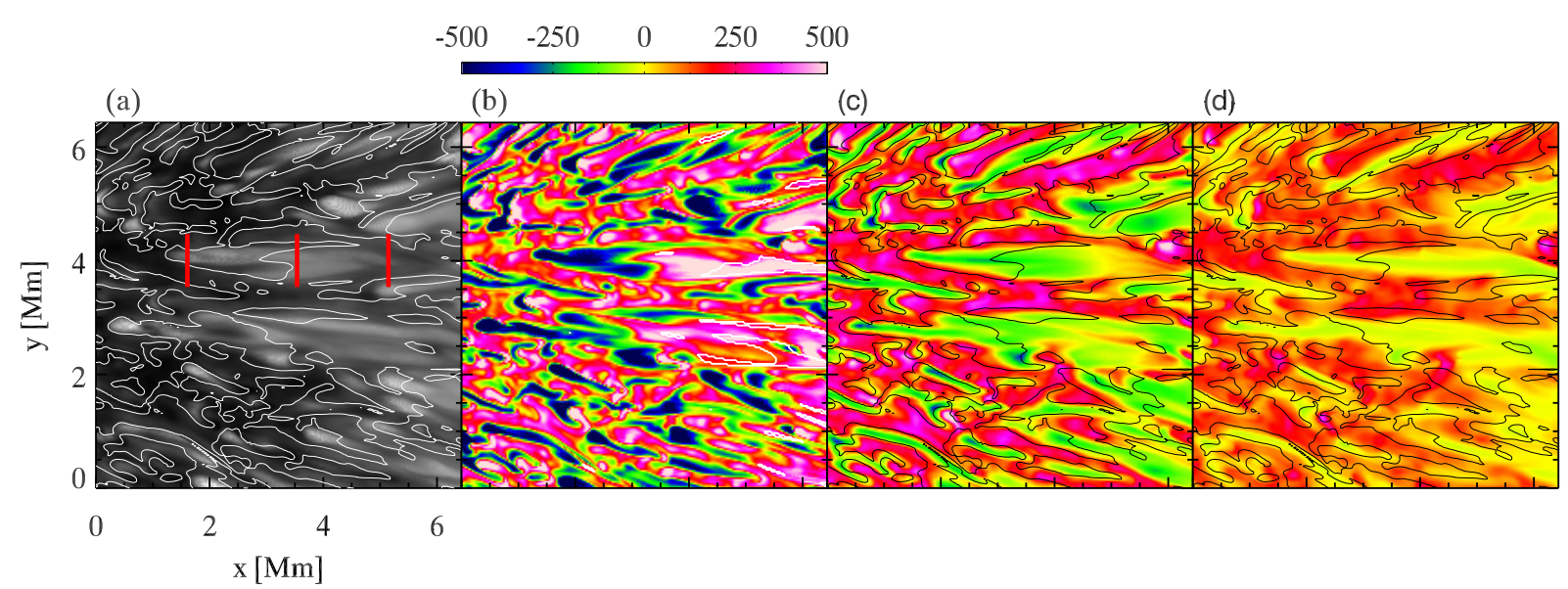

Fig. 18. Blowups of the area within the white boxes in Fig. 17. Contours in panels a); c); and d) encircles negative $(\Delta B / \Delta \log \tau)_{0.0,-0.9}$ and white contours in panel b) indicate areas with polarity opposite to that in the umbra. The three vertical bars in panel a) mark the locations of the vertical cuts displayed in Fig. 20.

Table 2. Comparison of spatially averaged $(\Delta B / \Delta \log \tau)$ values obtained from VTT/TIP-2, SOT/SP, and the MHD simulation in terms of the inner and outer halves of the penumbra.

\begin{tabular}{|c|c|c|c|}
\hline & VTT/TIP-2 & SOT/SP & MHD \\
\hline Inner & $\left(\frac{\Delta B}{\Delta \log \tau}\right)_{0.0,-2.3} \simeq-70 \mathrm{G} / \log \tau$ & $\begin{array}{l}\left(\frac{\Delta B}{\Delta \log \tau}\right)_{0.0,-0.9} \simeq-20 \mathrm{G} / \log \tau \\
\left(\frac{\Delta B}{\Delta \log \tau}\right)_{-0.9,-2.5} \simeq 73 \mathrm{G} / \log \tau\end{array}$ & $\begin{aligned}\left(\frac{\Delta B}{\Delta \log \tau}\right)_{0.0,-0.9} & \simeq 5 \mathrm{G} / \log \tau \\
\left(\frac{\Delta B}{\Delta \log \tau}\right)_{-0.9,-2.5} & \simeq 65 \mathrm{G} / \log \tau\end{aligned}$ \\
\hline Outer & $\left(\frac{\Delta B}{\Delta \log \tau}\right)_{0.0,-2.3} \simeq 9 \mathrm{G} / \log \tau$ & $\begin{array}{l}\left(\frac{\Delta B}{\Delta \log \tau}\right)_{0.0,-0.9} \simeq 137 \mathrm{G} / \log \tau \\
\left(\frac{\Delta B}{\Delta \log \tau}\right)_{-0.9,-2.5} \simeq 52 \mathrm{G} / \log \tau\end{array}$ & $\begin{array}{l}\left(\frac{\Delta B}{\Delta \log \tau}\right)_{0.0,-0.9} \simeq 172 \mathrm{G} / \log \tau \\
\left(\frac{\Delta B}{\Delta \log \tau}\right)_{-0.9,-2.5} \simeq 24 \mathrm{G} / \log \tau\end{array}$ \\
\hline
\end{tabular}

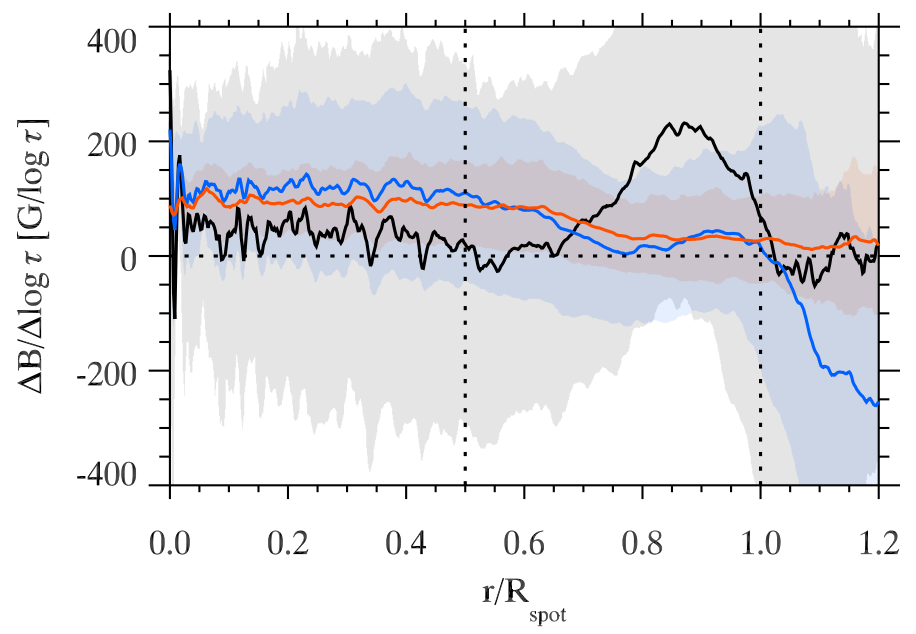

Fig. 19. Azimuthally averaged $(\Delta B / \Delta \log \tau)$ as a function of normalized radial distance $r / R_{\text {spot }}$ from the MHD simulation of a sunspot. Black, blue, and red curves depict $(\Delta B / \Delta \log \tau)_{0.0,-0.9},(\Delta B / \Delta \log \tau)_{-0.9,-2.5}$, and $(\Delta B / \Delta \log \tau)_{-2.5,-3.5}$, respectively. Shaded areas represent standard deviations. Dotted vertical lines indicate the umbra-penumbra boundary and the outer boundary of the sunspot.

the inner penumbra, as found in the SOT/SP and VTT/TIP-2 observations and in the MHD simulation.

\subsection{Effect of spatial resolution on $(\Delta B / \Delta \log \tau)$}

To understand the effect of the simplified model atmosphere used to fit the Stokes profiles observed with VTT/TIP-2, we inverted the Stokes profiles of the Si I $10827 \AA$ and Ca I $10833 \AA$ spectral lines synthesized along vertical rays passing through every $(x, y)$ grid point of the MHD simulation. The inversions start from the same set of initial parameters as were used to fit the VTT/TIP-2 data. One set of inversions is carried out at the native resolution of the MHD simulation, another after degrading the synthesized Stokes images by a point spread function (PSF) which has a Gaussian shape with FWHM of 1."0, close to the spatial resolution of the VTT/TIP-2 data presented in Sect. 2. In the second case, Stokes images are synthesized after binning the MHD atmosphere such that the resultant pixel size is equal to that in the VTT/TIP-2 data.

In Fig. 21, the radial dependence of $B($ at $\log \tau=0$ ) and $(\Delta B / \Delta \log \tau)_{0.0,-2.3}$, deduced from the inversions of the synthetic data are compared with the original $B$ and $(\Delta B / \Delta \log \tau)_{0.0,-2.3}$ values obtained from the MHD simulation. From the sunspot center to half of the sunspot radius, $B$ is reproduced reasonably by the inversions of synthesized Stokes profiles, both at native and degraded spatial resolution of the MHD simulation. The inversions tend to overestimate both $B$ and its vertical gradient. In the outer half of the sunspot $B$ is underestimated by $\sim 200 \mathrm{G}$ when we perform inversions of Stokes profiles synthesized in native spatial resolution. The inversion of spatially degraded Stokes profiles results in a further underestimate of $B$ by up to $\sim 1000 \mathrm{G}$, mainly as a result of signal cancellation in unresolved structures. Between $r / R_{\text {spot }} \simeq 0.20$ and $r / R_{\text {spot }} \simeq 0.70$ the 


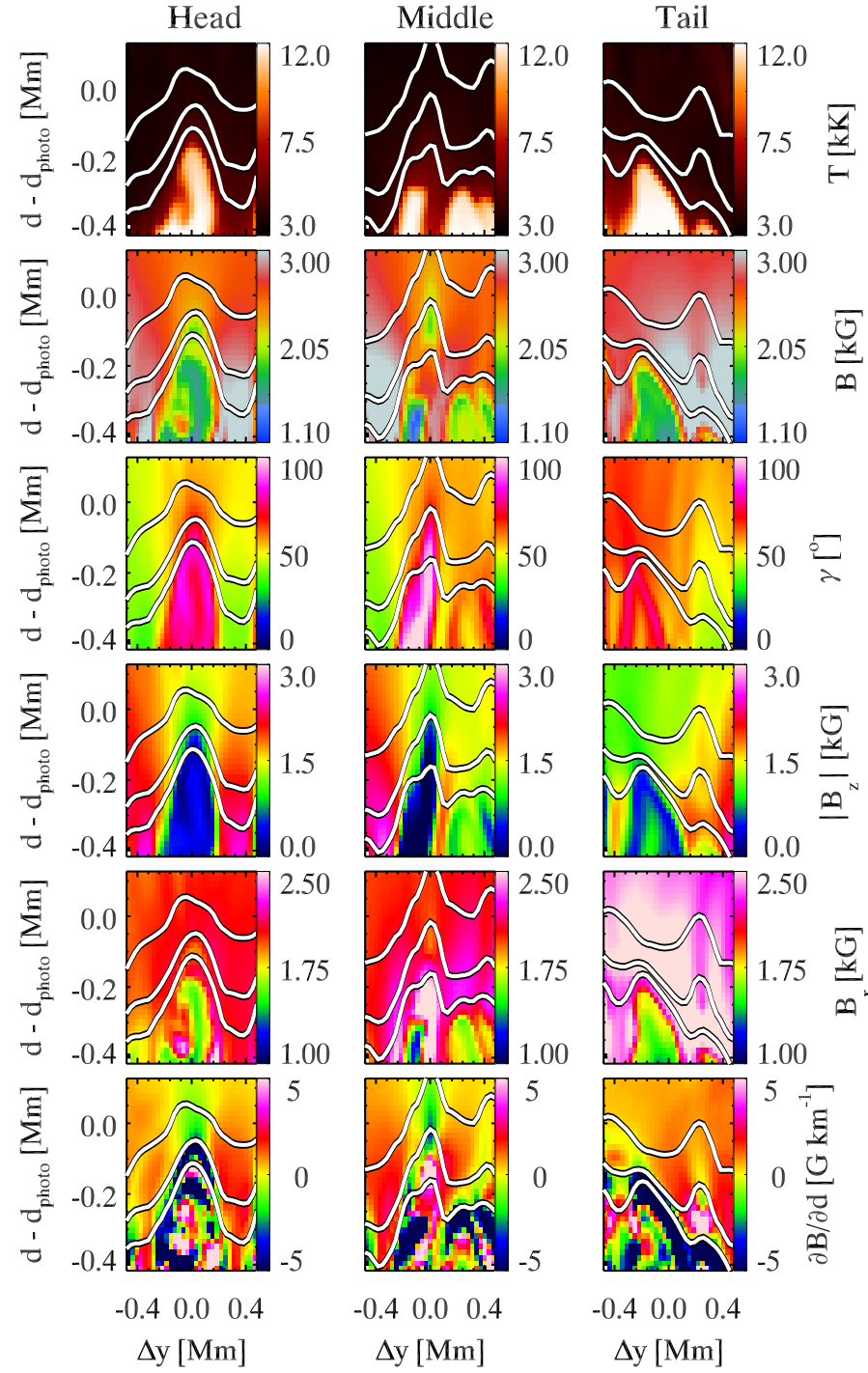

Fig. 20. Geometrical depth stratification of various physical parameters perpendicular to the axis of a filament in the MHD simulation. The locations of the plotted cuts are indicated by red bars in Fig. 18a. Panels in the first, second, and third column represent the head, middle, and tail of the filament, respectively. From top to bottom (for each column): $T, B, \gamma,\left|B_{\mathrm{z}}\right|, B_{\mathrm{r}}$, and $\partial B / \partial d$ are plotted. Lower, middle, and upper white curves in all panels show $\log \tau=0.0,-0.9,-2.5$ levels, respectively.

inversions with the simplified model at native resolution overestimate $(\Delta B / \Delta \log \tau)_{0.0,-2.3}$, while between $r / R_{\text {spot }} \simeq 0.75$ and $r / R_{\text {spot }}=1.0$ they underestimate $(\Delta B / \Delta \log \tau)_{0.0,-2.3}$, but displays the correct sign. The inversion results of the spatially degraded Stokes profiles show a qualitative difference to the true gradient in the model, leading to negative $(\Delta B / \Delta \log \tau)_{0.0,-2.3}$ in the outer penumbra. The main result from the VTT/TIP-2 data, namely the dominant presence of negative $(\Delta B / \Delta \log \tau)_{0.0,-2.3}$ in parts of the penumbra, is reproduced, although the negative values of $(\Delta B / \Delta \log \tau)_{0.0,-2.3}$ occur in the outer part of the penumbra in the MHD simulation, while they are located in the inner penumbra in the observations.

This experiment demonstrates that a lower spatial resolution can lead to apparent negative $(\Delta B / \Delta \log \tau)$ in the penumbra. One possibility is that unresolved opposite polarity patches in the penumbra could significantly influence $(\Delta B / \Delta \log \tau)$ deduced from observations, especially if the population of opposite polarity patches changes with height in the photosphere.
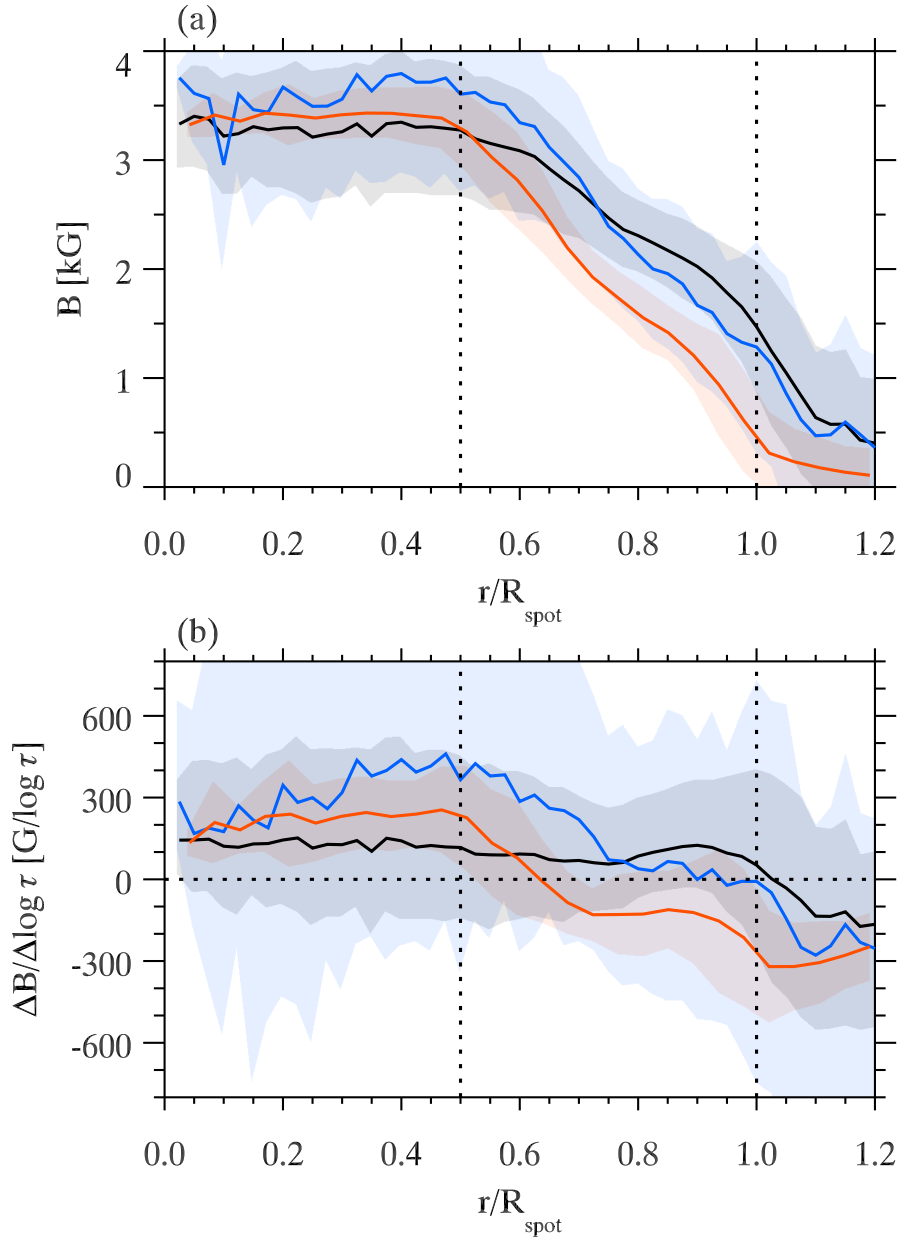

Fig. 21. Radial dependence of $B$ at $\log \tau=0$ (panel a)) and $(\Delta B / \Delta \log \tau)_{0.0,-2.3}($ panel b)) from the MHD simulation. Black curves are estimated directly from the MHD simulation and blue curves are the results of inversions of synthesized Stokes profiles from the MHD simulation at native resolution. Red curves are from inversions of synthesized Stokes profiles from the MHD simulation degraded to a spatial resolution of $\sim 1$ ". 0 . Shaded areas represent standard deviation. The dotted vertical lines in both panels indicate the umbra-penumbra boundary and the outer boundary of the sunspot.

\section{Opposite polarity patches in penumbrae}

In this section we analyze the distribution of opposite polarity patches at different $\log \tau$ surfaces in the MHD simulation and in the SOT/SP observations in order to understand the relation between opposite polarity patches and the presence of negative $(\Delta B / \Delta \log \tau)$.

The positions of patches with polarity opposite to that in the umbra at three $\log \tau$ levels $(0.0,-0.9$, and -2.5$)$ in the MHD simulation are shown in Fig. 22. At $\log \tau=0$ the opposite polarity patches appear at almost all radial distances in the penumbra with the number of patches increasing towards the outer penumbra. At $\log \tau=-0.9$ and $\log \tau=-2.5$ the opposite polarity patches are mainly restricted to the middle to outer penumbra and to the outer penumbra, respectively. The positions of the opposite polarity patches in the SOT/SP data are consistent with the MHD simulation (see Fig. 23). The radial distribution of opposite polarity flux $B_{z}^{\text {opp }}$ as a fraction of the net flux $\left|B_{z}\right|$ is plotted in Fig. 24. In the SOT/SP observations, opposite polarity flux appears even in the inner penumbra at $\log \tau=0$, and reaches a fraction of up to $\sim 55 \%$ of the total flux at the outer 
(a)

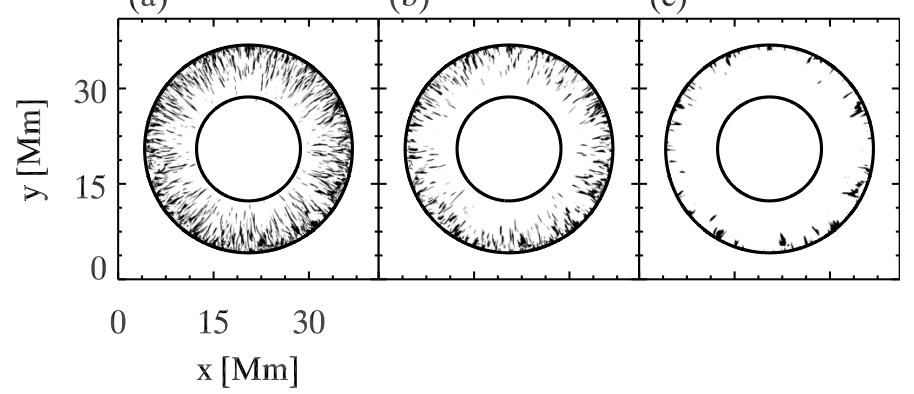

Fig. 22. Maps of opposite polarity patches from the MHD simulation of a sunspot. Black areas indicate positions of opposite magnetic polarity. Panels a)-c) correspond to $\log \tau=0, \log \tau=-0.9$, and $\log \tau=-2.5$, respectively. Circles in all panels represent the umbra-penumbra boundary and the outer boundary of the sunspot.

(a)

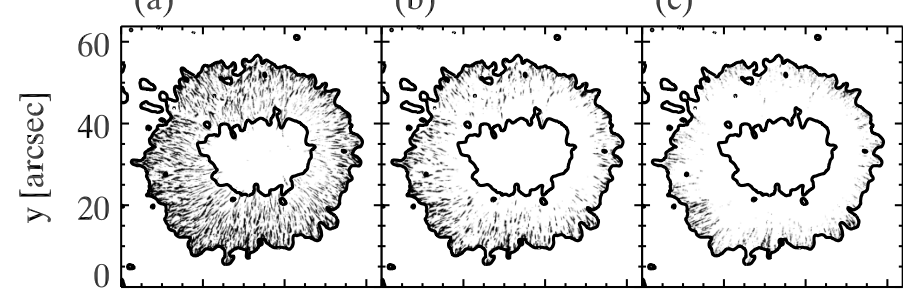

$\begin{array}{llll}0 & 20 & 40 & 60\end{array}$

$\mathrm{x}[\operatorname{arcsec}]$

Fig. 23. Same as Fig. 22 but for SOT/SP observations.

boundary of the penumbra. The MHD simulation shows $\sim 47 \%$ flux with opposite polarity at the outer boundary of the penumbra, but starting at a larger $r / R_{\text {spot }}$ value. At $\log \tau=-0.9$ the SOT/SP data have only $\sim 15 \%$ flux with opposite polarity at the outer boundary of the penumbra, whereas it amounts to $\sim 30 \%$ in the MHD simulation. Approximately $8 \%$ of the flux has the opposite polarity in the outer penumbra at $\log \tau=-2.5$ in both the SOT/SP data and the MHD simulation. When the MHD simulation is degraded to 1 ". 0 only a small fraction of the opposite polarity flux survives (see panel c of Fig. 24). With degraded spatial resolution a higher number of pixels with negative $(\Delta B / \Delta \log \tau)$ is found in the penumbra than in the native resolution of the MHD simulation (see panel d of Fig. 24). In the umbra not a single pixel is found with negative values of $(\Delta B / \Delta \log \tau)$ in the degraded spatial resolution data, but almost $\sim 30 \%$ of the pixels have negative values of $(\Delta B / \Delta \log \tau)$ in the native resolution presumably corresponding to umbral-dots (Schüssler \& Vögler 2006; Riethmüller et al. 2008, 2013). In summary, the low spatial resolution hides opposite polarity patches in the sunspot. However, it causes many pixels with $(\Delta B / \Delta \log \tau)<0$, hence, it also produces $(\Delta B / \Delta \log \tau)<0$ in the azimuthal averages.

\section{Discussion}

We determined the vertical gradient of the magnetic field strength throughout sunspots. From the VTT/TIP-2 observations we found that $B$ increases at a rate of approximately $1.3 \mathrm{G} \mathrm{km}^{-1}$ with geometrical depth above $\log \tau=0.0$ in the umbra. In the SOT/SP data we found that $B$ increases with depth at an average rate of $\sim 1.4 \mathrm{G} \mathrm{km}^{-1}$ in the umbra. Here we present the gradient in terms of geometrical depth in order to facilitate comparisons with earlier studies. Similar values have been found by Borrero \& Ichimoto (2011) and Tiwari et al. (2015) based
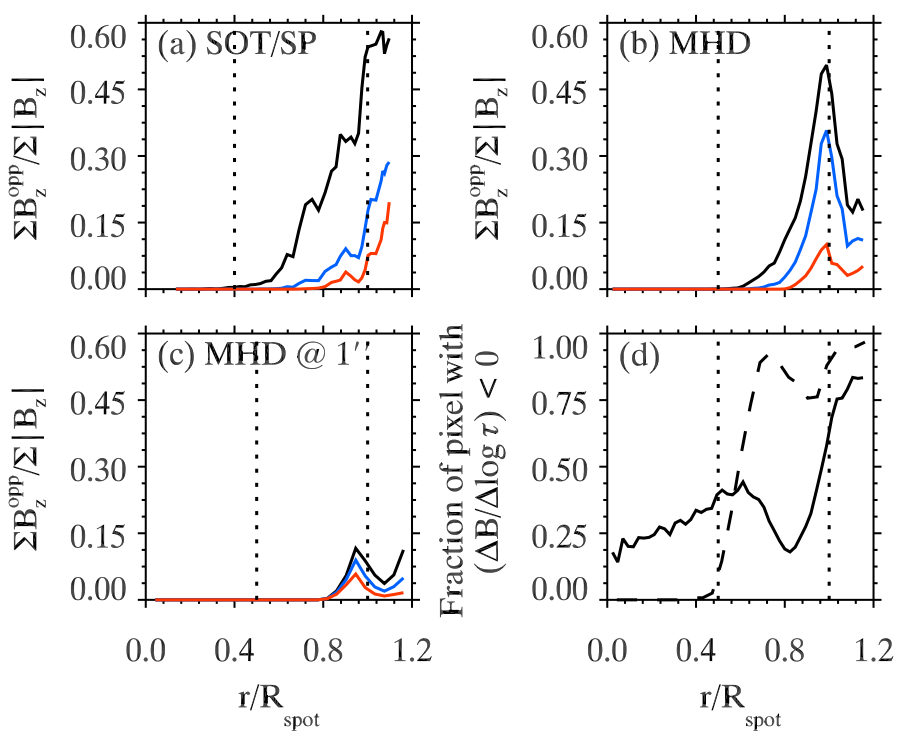

Fig. 24. Panel a) shows the radial distribution of opposite polarity flux $B_{z}^{\text {opp }}$ as a fraction of the net flux $\left|B_{z}\right|$ from the SOT/SP observations. Panel b) displays the radial distribution of opposite polarity flux for the MHD simulation. Panel c) corresponds to opposite flux from inversions of synthesized Stokes profiles from the MHD simulation which was degraded with a Gaussian of 1".0 width. Black, blue, and red curves correspond to $\log \tau=0.0, \log \tau=-0.9$, and $\log \tau=-2.5$, respectively. Solid and dashed curves in panel (d) display the numbers of pixels where $(\Delta B / \Delta \log \tau)_{0.0,-2.3}<0$ at native and degraded spatial resolution in the MHD simulation, respectively. Dotted vertical lines in all the panels indicate the umbra-penumbra boundary and the outer boundary of the sunspot.

on Hinode SOT/SP data and by Sánchez Cuberes et al. (2005) from VTT/TIP data. Mathew et al. (2003) report values around $4 \mathrm{G} \mathrm{km}^{-1}$ and Westendorp Plaza et al. (2001) found $2.5 \mathrm{G} \mathrm{km}^{-1}$. We found that $B$ increases rapidly with depth in the lower atmosphere, while the gradient becomes flatter in the upper atmosphere above $\log \tau=-0.9$ in the SOT/SP data. This might explain the difference in the value found by Mathew et al. (2003), who obtained the gradient from the inversion of the Fe I $1.56 \mu \mathrm{m}$ lines that form very deeply in the photosphere.

The vertical gradient of the magnetic field strength in the penumbra reported in the present work displays large fluctuations, changing its sign even on small scales. In the gradient maps of the penumbra derived from the SOT/SP observations, $(\Delta B / \Delta \log \tau)$ is always positive in spines. The gradient in the penumbral filaments has a more complex structure: the lower layer of the inner half of the filaments (i.e., the part of a filament closer to the umbra) has a positive $(\Delta B / \Delta \log \tau)$ which is surrounded by a negative $(\Delta B / \Delta \log \tau)$ at the sides. In the upper layer of the inner half of the filaments it only has a negative $(\Delta B / \Delta \log \tau)$ that is strongest along the central ridge of the filaments. The outer half of the filament has a positive $(\Delta B / \Delta \log \tau)$ both in the lower and upper layers. The positive $(\Delta B / \Delta \log \tau)$ in the tail can be explained by the fact that the horizontal field of the filament, which is returning to the surface, is stronger than the spine's field covering the filaments from the top (Tiwari et al. 2013). The decrease in the number and strength of spines with $r / R_{\text {spot }}$ probably also contributes. The structure of the gradient in the body of filaments close to the head can be explained by a penumbral model presented in Fig. 25 (adopted from Zakharov et al. 2008). The vertical structure of a penumbral filament is assumed to be a semicircular horizontal cylinder containing a nearly horizontal magnetic field. 


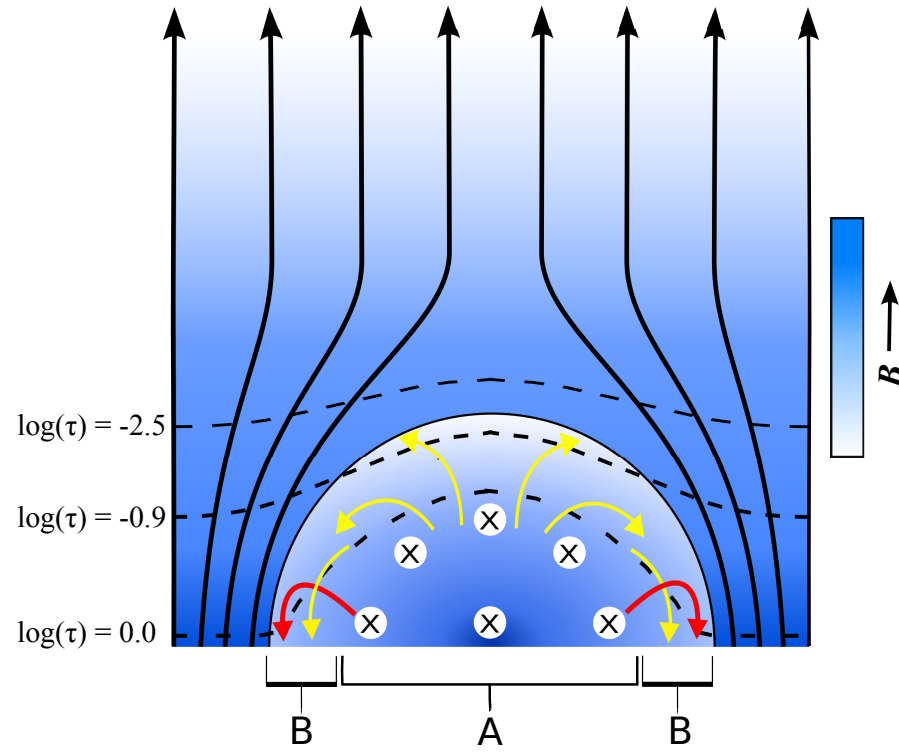

Fig. 25. Schematic view of the vertical structure of the magnetic field in penumbral filaments following Zakharov et al. (2008). Depicted is a plane perpendicular to the filament. The filament is represented by the semicircular area. The crosses in the circular white areas indicate the direction of the magnetic field in the penumbral filament, which is perpendicular to the plane of the drawing. Yellow arrows indicate the overturning convection within the filament and red arrows depict the field reversal at the edges of the filaments, which is caused by the convective flow through advection of the filament's field. Only the projected component of the field, which is directed mainly into the page even for the red arrows, is plotted. The background magnetic field (spines) is indicated by nearly vertical black arrows. Dashed lines show different $\log \tau$ levels. Lighter and darker shades of blue correspond to weaker and stronger $B$, respectively.

The filament is surrounded by a more vertical background magnetic field (spines) on the sides that closes above the filament. The background field forms spines with $B$ gradually decreasing with height. This model assumes that the semicircular filament has its maximum magnetic field strength at its center, which gradually decreases towards the boundary between the spines and the filament. The $\log \tau=0.0$ level samples the whole width of the filament (shown by region $B+A+B$ in Fig. 25), whereas the $\log \tau=-0.9$ level samples only the central part of the filament's width (region A). Finally, the $\log \tau=-2.5$ level sees the field only from the spines. In region A the field strength decreases between $\log \tau=0.0$ and $\log \tau=-0.9$, hence $(\Delta B / \Delta \log \tau)_{0.0,-0.9}$ is positive. In region $\mathrm{B}(\Delta B / \Delta \log \tau)_{0.0,-0.9}$ is negative because the $\log \tau=0.0$ level probes the filament, whereas the $\log \tau=-0.9$ level samples the stronger field from the spines. The value of $(\Delta B / \Delta \log \tau)_{-0.9,-2.5}<0$ in region $A$ because the $\log \tau=-0.9$ level is still within the body of the filament, whereas the $\log \tau=-2.5$ level is above the filament where the stronger field from the spines closes. In summary, the expanding and closing magnetic field from the spines above the filament causes the negative $(\Delta B / \Delta \log \tau)$ in the inner part of penumbral filaments. It is worthwile to mention here that

In the MHD simulation $(\Delta B / \Delta \log \tau)$ is negative around the heads of filaments in both layers. The sign of $(\Delta B / \Delta \log \tau)_{0.0,-0.9}$ is positive in the tail of filaments, whereas $(\Delta B / \Delta \log \tau)_{-0.9,-2.5}$ is negative there. The gradient maps thus reveals that there are differences in the details of magnetic field structure of the penumbral filaments between the MHD simulation and the observations.
After producing azimuthal averages, our results turn out to be rather different from the results published previously (Westendorp Plaza et al. 2001; Mathew et al. 2003; Sánchez Cuberes et al. 2005; Balthasar \& Gömöry 2008; Borrero \& Ichimoto 2011). In the VTT/TIP-2 data, where we assume that $B$ varies linearly with $\log \tau$, we found that $B$ decreases with depth from the umbra-penumbra boundary to $r \simeq 0.75 R_{\text {spot }}$. Between $r \simeq 0.75 R_{\text {spot }}$ and $r / R_{\text {spot }}=1.0$ $B$ increases with depth. The SOT/SP observations exhibit a similar profile of $(\Delta B / \Delta \log \tau)$ in the lower layers. In the upper atmosphere $B$ increases on average with depth at all radial distances in the penumbra. The value of $(\Delta B / \Delta \log \tau)_{0.0,-0.9}$ estimated from the MHD simulation shows good qualitative similarities with the SOT/SP data in the lower layers, although $(\Delta B / \Delta \log \tau)_{0.0,-0.9}$ in the MHD simulation is mostly close to zero (slightly negative) in the inner penumbra. The average radial profiles of $(\Delta B / \Delta \log \tau)$ found in the present study with VTT/TIP-2 and SOT/SP observations disagree with earlier observational studies. Westendorp Plaza et al. (2001) and Borrero \& Ichimoto (2011) found that $B$ increases with depth in the inner half radius of the sunspot. In the outer half radius the field decreases with depth. In contrast, Mathew et al. (2003) and Sánchez Cuberes et al. (2005) found that $B$ increases with depth everywhere within the sunspot's visible boundary. An observation of a reversed gradient in the vertical magnetic field component in the penumbra has been reported by Balthasar et al. (2013), based on the difference between the magnetic field inferred from the Fe I $10783 \AA$ and Si I $10786 \AA$ lines.

The expansion of the sunspot's magnetic field due to the decreasing gas pressure, $(\partial P / \partial d)>0$, should always produce a positive $(\Delta B / \Delta \log \tau)$ on a global scale. This is in seeming contradiction with the azimuthally averaged radial profiles of $(\Delta B / \Delta \log \tau)_{0.0,-0.9}$ found in the SOT/SP observations and the MHD simulation where $(\Delta B / \Delta \log \tau)_{0.0,-0.9}$ is negative in the inner penumbra. Highly corrugated optical depth surfaces, sampling the atmosphere at different geometrical depths in different parts of the penumbra, can cause the observed negative gradient in the azimuthal averages. In the penumbral filaments, the $\log \tau$ surfaces are elevated compared to the spines (see Figs. 20 and 25). In the spines the magnetic field strength changes gradually in contrast to the filament exhibiting an abrupt change in the magnetic field strength (from filament to the overlying field of the expanding spines) between two $\log \tau$ surfaces. The scenario described above leads to a negative $(\Delta B / \Delta \log \tau)_{0.0,-0.9}$ with higher magnitude in and above the filaments compared to the positive $(\Delta B / \Delta \log \tau)_{0.0,-0.9}$ in the spines. The negative $(\Delta B / \Delta \log \tau)_{0.0,-0.9}$ is only seen around the heads of the filaments. A higher density of filament heads at a given $r / R_{\text {spot }}$ leads to a higher probability of negative values for the azimuthal averages of $(\Delta B / \Delta \log \tau)_{0.0,-0.9}$. This is the case in the inner penumbra, compared to the middle and outer penumbra.

The statement that the highly corrugated $\log \tau$ surfaces cause the negative gradients in the azimuthal averages is supported by the MHD simulation. The azimuthally averaged gradient with respect to the geometrical scale, $\partial B / \partial d$, from the MHD simulation does not show negative values in the inner penumbra above $\log \tau=0$. When $(\Delta B / \Delta \log \tau)$ is examined then only the MHD simulations show a negative or close to zero vertical gradient of the magnetic field in the inner penumbra.

An underestimation of $B$ in deeper layers could also contribute to the observed negative gradient in the inner penumbra. Underestimation of $B$ can be caused by a cancellation of a polarized signal stemming from unresolved 
opposite polarity areas. The presence of opposite polarity patches in sunspot penumbrae has been observed by Scharmer et al. (2013), Ruiz Cobo \& Asensio Ramos (2013), Franz \& Schlichenmaier (2013), Tiwari et al. (2013), and van Noort et al. (2013). Ruiz Cobo \& Asensio Ramos (2013) and Franz \& Schlichenmaier (2013) have found the opposite polarity patches in the middle and outer penumbra in SOT/SP observations while Scharmer et al. (2013) found thinner and elongated lanes of opposite polarity everywhere in the penumbra in higher resolution SST/CRISP observations. Recent 3D MHD simulations of a sunspot by Rempel (2012) (cf. Rempel et al. 2009) also exhibit opposite polarity patches in the penumbra. These patches of opposite polarities are thought to be produced by advection of the field by the overturning convection in penumbrae. In recent years many observational evidences for overturning convection in penumbrae has been reported (Joshi et al. 2011; Scharmer et al. 2011; Tiwari et al. 2013; Esteban Pozuelo et al. 2015).

Our analysis of opposite polarity patches in the SOT/SP observations and the MHD simulation indicates that the flux associated with opposite polarity patches increases from the inner to the outer penumbra (see also Fig. 13 in Rempel 2012). Both the MHD simulation and the SOT/SP observations reveals the presence of opposite polarity patches even in the higher part of the atmosphere, i.e., up to $\log \tau=-2.3$. The distribution of opposite polarity patches in the penumbra observed with SOT/SP is qualitatively consistent with that of the MHD simulation (see Figs. 23 and 24), although there is a quantitative difference in the flux associated with opposite polarity patches in the SOT/SP observations and the MHD simulation. In the upper layers opposite polarity patches are restricted to the middle and outer penumbra both in the SOT/SP observations and the MHD simulation. These opposite polarity patches are of two kinds. The first are narrower and elongated lanes of opposite polarity at the edges of the inner part of penumbral filaments (see Fig. 25) at the locations of downflow lanes, which are the lateral components of overturning convection (Scharmer et al. 2013; Tiwari et al. 2013). The second kind of patches found at the tails of the penumbral filaments are bigger in size (Franz \& Schlichenmaier 2013; Ruiz Cobo \& Asensio Ramos 2013; Scharmer et al. 2013; Tiwari et al. 2013; van Noort et al. 2013) and are associated with downflows at the tails.

Our experiment, estimating $(\Delta B / \Delta \log \tau)_{0.0,-2.3}$ from the degraded spatial resolution (1".0) MHD simulation shows that at this resolution opposite polarity patches disappear almost completely. At the same time a significant increase in the number of pixels with negative $(\Delta B / \Delta \log \tau)_{0.0,-2.3}$ occurs in the penumbra. This behavior reverses in the umbra where in the native resolution $\sim 30 \%$ of the pixels have negative $(\Delta B / \Delta \log \tau)_{0.0,-2.3}$ that disappear completely in the degraded resolution data. As discussed above, we have found more flux with opposite polarity in the lower layers than in the upper layers, thus lower spatial resolution causes more cancellation in the polarized signal from deeper layers and produces negative $(\Delta B / \Delta \log \tau)_{0.0,-2.3}$.

As far as the SOT/SP observations are concerned, a significant amount of flux is associated with opposite polarity patches, but it is still possible that the narrow and elongated opposite polarity lanes at the edges of the penumbral filaments are not completely resolved. In this case it is possible that some fraction of the negative gradient originates from unresolved opposite polarity patches. However, the radial distribution of opposite polarity patches and the number of pixels with negative values of $(\Delta B / \Delta \log \tau)_{0.0,-2.3}$ are rather different. This suggests that the main cause of the observed negative $(\Delta B / \Delta \log \tau)_{0.0,-2.3}$ in the azimuthal averages are the highly corrugated iso- $\tau$ surfaces.

The presence of a magnetic canopy outside the visible boundary of the sunspot (Solanki et al. 1992, 1994,1999; Lites et al. 1993; Adams et al. 1994; Skumanich et al. 1994; Keppens \& Martinez Pillet 1996; Rueedi et al. 1998), where the canopy base rises with distance from the sunspot, can be concluded from the SOT/SP observations. This is also consistent with the results from the MHD simulation and is in line with the results of Tiwari et al. (2015). The vertical gradient of $B$ turns out to be a useful quantity for identifying or detecting magnetic canopies.

An important result of this study is that on large scales the magnetic canopy only exists at and outside the visible boundary of the sunspot $\left(r / R_{\text {spot }}>0.9\right)$. We do not see a canopy structure in the middle penumbra caused by the expansion of field from the umbra or the inner penumbra as reported by Westendorp Plaza et al. (2001) and Borrero \& Ichimoto (2011). Instead, we found local patches in the penumbra where the magnetic field strength decreases with optical depth caused by the stronger field in the spines closing above the filaments (see Fig. 25). The corrugation effect of the $\tau$ surfaces leads to negative $(\Delta B / \Delta \log \tau)$ in the inner penumbra in the lower atmosphere in the azimuthal average. In addition to the corrugation of the $\tau$ surfaces, the effect of unresolved, deep-lying opposite polarity patches can also lead to negative $(\Delta B / \Delta \log \tau)$ patches in the penumbra.

Acknowledgements. The German Vacuum Tower Telescope is operated by the Kiepenheuer-Institut für Sonnenphysik at the Spanish Observatorio del Teide of the Instituto de Astrofísica de Canarias (IAC). Hinode is a Japanese mission developed and launched by ISAS/JAXA, with NAOJ as domestic partner and NASA and STFC (UK) as international partners. It is operated by these agencies in co-operation with ESA and NSC (Norway). We thank M. Rempel for providing the MHD simulations. J.J. acknowledges a Ph.D. fellowship of the International Max Planck Research School on Physical Processes in the Solar System and Beyond (IMPRS). This work was partly supported by the BK21 plus program through the National Research Foundation (NRF) funded by the Ministry of Education of Korea. S.K.T. is supported by an appointment to the NASA Postdoctoral Program at the NASA Marshall Space Flight Center, administered by Universities Space Research Association under contract with NASA.

\section{References}

Adams, M., Solanki, S. K., Hagyard, M. J., \& Moore, R. L. 1994, in Cool Stars, Stellar Systems, and the Sun, ed. J.-P. Caillault, ASP Conf. Ser., 64, 342

Balthasar, H., \& Gömöry, P. 2008, A\&A, 488, 1085

Balthasar, H., Beck, C., Gömöry, P., et al. 2013, Central Eur. Astrophys. Bull., 37,435

Bard, S., \& Carlsson, M. 2008, ApJ, 682, 1376

Borrero, J. M., \& Ichimoto, K. 2011, Liv. Rev. Sol. Phys., 8, 4

Chapman, G. A. 1979, ApJ, 232, 923

Collados, M. 1999, in Third Advances in Solar Physics Euroconference: Magnetic Fields and Oscillations, eds. B. Schmieder, A. Hofmann, \& J. Staude, ASP Conf. Ser., 184, 3

Collados, M. V. 2003, in Polarimetry in Astronomy, ed. S. Fineschi, SPIE Conf. Ser., 4843, 55

Collados, M., Lagg, A., Díaz Garcí A, J. J., et al. 2007, in The Physics of Chromospheric Plasmas, eds. P. Heinzel, I. Dorotovič, \& R. J. Rutten, ASP Conf. Ser., 368, 611

Cuperman, S., Li, J., \& Semel, M. 1992, A\&A, 265, 296

Esteban Pozuelo, S., Bellot Rubio, L. R., \& de la Cruz Rodríguez, J. 2015, ApJ, 803,93

Franz, M., \& Schlichenmaier, R. 2013, A\&A, 550, A97

Frutiger, C., Solanki, S. K., Fligge, M., \& Bruls, J. H. M. J. 1999, in Polarization, eds. K. N. Nagendra, \& J. O. Stenflo, Astrophys. Space Sci. Lib., 243, 281

Frutiger, C., Solanki, S. K., Fligge, M., \& Bruls, J. H. M. J. 2000, A\&A, 358, 1109

Grevesse, N., \& Sauval, A. J. 1998, Space Sci. Rev., 85, 161

Ichimoto, K., Tsuneta, S., Suematsu, Y., et al. 2008, A\&A, 481, L9 
Joshi, J. 2014, Ph.D. Thesis, Braunschweig University, Germany

Joshi, J., Pietarila, A., Hirzberger, J., et al. 2011, ApJ, 734, L18

Keppens, R., \& Martinez Pillet, V. 1996, A\&A, 316, 229

Kuckein, C., Martínez Pillet, V., \& Centeno, R. 2012, A\&A, 539, A131

Lites, B. W., Elmore, D. F., Seagraves, P., \& Skumanich, A. P. 1993, ApJ, 418, 928

Livingston, W., \& Wallace, L. 1991, An atlas of the solar spectrum in the infrared from 1850 to $9000 \mathrm{~cm}-1,1.1$ to 5.4 micrometer) (Tucson: National Solar observatory)

Mathew, S. K., Lagg, A., Solanki, S. K., et al. 2003, A\&A, 410, 695

Mathew, S. K., Martínez Pillet, V., Solanki, S. K., \& Krivova, N. A. 2007, A\&A 465,291

Orozco Suarez, D., Lagg, A., \& Solanki, S. K. 2005, in Chromospheric and Coronal Magnetic Fields, eds. D. E. Innes, A. Lagg, \& S. A. Solanki, ESA SP, 596

Rempel, M. 2011, ApJ, 729, 5

Rempel, M. 2012, ApJ, 750, 62

Rempel, M., Schüssler, M., Cameron, R. H., \& Knölker, M. 2009, Science, 325, 171

Riethmüller, T. L., Solanki, S. K., \& Lagg, A. 2008, ApJ, 678, L157

Riethmüller, T. L., Solanki, S. K., van Noort, M., \& Tiwari, S. K. 2013, A\&A, 554, A53

Rueedi, I., Solanki, S. K., Keller, C. U., \& Frutiger, C. 1998, A\&A, 338, 1089

Ruiz Cobo, B., \& Asensio Ramos, A. 2013, A\&A, 549, L4

Sakurai, T., Makita, M., \& Shibasaki, K. 1985, Max Planck Institut fur Astrophysik Report, 212, 312

Sánchez Cuberes, M., Puschmann, K. G., \& Wiehr, E. 2005, A\&A, 440, 345

Scharmer, G. B., Henriques, V. M. J., Kiselman, D., \& de la Cruz Rodríguez, J. 2011, Science, 333, 316
Scharmer, G. B., de la Cruz Rodriguez, J., Sütterlin, P., \& Henriques, V. M. J. 2013, A\&A, 553, A63

Schüssler, M., \& Vögler, A. 2006, ApJ, 641, L73

Shchukina, N., Sukhorukov, A., \& Trujillo Bueno, J. 2012, ApJ, 755, 176

Shi, J. R., Gehren, T., Butler, K., Mashonkina, L. I., \& Zhao, G. 2008, A\&A, 486, 303

Shimizu, T., Nagata, S., Tsuneta, S., et al. 2008, Sol. Phys., 249, 221

Skumanich, A., Lites, B. W., \& Martínez Pillet, V. 1994, in Solar Surface Magnetism, eds. R. J. Rutten \& C. J. Schrijver (Dordrecht: Kluwer Academic Publishers), 99

Solanki, S. K. 1987, Ph.D. Thesis, No. 8309, ETH, Zürich, Switzerland

Solanki, S. K. 2003, A\&ARv, 11, 153

Solanki, S. K., Rueedi, I., \& Livingston, W. 1992, A\&A, 263, 339

Solanki, S. K., Montavon, C. A. P., \& Livingston, W. 1994, A\&A, 283, 221

Solanki, S. K., Finsterle, W., Rüedi, I., \& Livingston, W. 1999, A\&A, 347, L27

Suematsu, Y., Tsuneta, S., Ichimoto, K., et al. 2008, Sol. Phys., 249, 197

Tiwari, S. K., van Noort, M., Lagg, A., \& Solanki, S. K. 2013, A\&A, 557, A25

Tiwari, S. K., van Noort, M., Solanki, S. K., \& Lagg, A. 2015, A\&A, 583, A119

Tsuneta, S., Ichimoto, K., Katsukawa, Y., et al. 2008, Sol. Phys., 249, 167

van Noort, M. 2012, A\&A, 548, A5

van Noort, M., Lagg, A., Tiwari, S. K., \& Solanki, S. K. 2013, A\&A, 557, A24

Wallace, L., Hinkle, K., \& Livingston, W. C. 1993, An atlas of the photospheric spectrum from 8900 to $13600 \mathrm{~cm}^{-1}$ (7350 to $11230 \AA$ A), Tech. Rep. (Tucson: National Solar Observatory)

Westendorp Plaza, C., del Toro Iniesta, J. C., Ruiz Cobo, B., et al. 2001, ApJ, 547, 1130

Wilkinson, L. K., Emslie, G. A., \& Gary, G. A. 1989, Sol. Phys., 119, 77

Zakharov, V., Hirzberger, J., Riethmüller, T. L., Solanki, S. K., \& Kobel, P. 2008, A\&A, 488, L17 